\title{
Paxillin Is Required for Proper Spinal Motor Axon Growth into the Limb
}

\author{
Wan-Ling Tsai, ${ }^{1 *}$ Chih-Ju Chang, ${ }^{2,3,4 *}$ Chih-Yang Wang, ${ }^{5}{ }^{\circledR}$ Tsung-I Hsu,,${ }^{6,78}$ Ming-Yuan Chang, ${ }^{9}{ }^{\circ}$ Yi-Hsin Wu, ${ }^{6}$ \\ Pei-Shan Chang, ${ }^{6,10}$ Kai-Lun Lin, ${ }^{6,10}$ Jian-Ying Chuang, ${ }^{6,7,8}{ }^{-}$Artur Kania, ${ }^{11,12}$ and ${ }^{-}$Tzu-Jen Kao ${ }^{6,7,8}$ \\ ${ }^{1} \mathrm{Ph}$.D. Program for Neural Regenerative Medicine, College of Medical Science and Technology, Taipei Medical University and National Health Research \\ Institutes, Taipei 110, Taiwan, ${ }^{2}$ Department of Neurosurgery, Cathay General Hospital, Taipei 110, Taiwan, ${ }^{3}$ School of Medicine, Fu Jen Catholic University, \\ New Taipei 242, Taiwan, ${ }^{4}$ Department of Mechanical Engineering, National Central University, Taoyuan 320, Taiwan, ${ }^{5}$ Graduate Institute of Cancer \\ Biology and Drug Discovery, College of Medical Science and Technology, Taipei Medical University, Taipei 110, Taiwan, ${ }^{6} \mathrm{Graduate}$ Institute of \\ Neural Regenerative Medicine, College of Medical Science and Technology, Taipei Medical University, Taipei 110, Taiwan, ${ }^{7}$ Research Center of \\ Neuroscience, Taipei Medical University, Taipei 110, Taiwan, ${ }^{8}$ TMU Research Center of Cancer Translational Medicine, Taipei Medical University, \\ Taipei 110, Taiwan, ${ }^{9}$ Division of Neurosurgery, Department of Surgery, Min-Sheng General Hospital, Taoyuan 330, Taiwan, ${ }^{10}$ School of Pharmacy, \\ Taipei Medical University, Taipei 110, Taiwan, ${ }^{11}$ Institut de recherches cliniques de Montréal, Montréal, Quebec H2W 1R7, Canada, and \\ ${ }^{12}$ Department of Anatomy and Cell Biology, Division of Experimental Medicine, McGill University, Montréal, Quebec H3A 2B2, Canada
}

To assemble the functional circuits of the nervous system, the neuronal axonal growth cones must be precisely guided to their proper targets, which can be achieved through cell-surface guidance receptor activation by ligand binding in the periphery. We investigated the function of paxillin, a focal adhesion protein, as an essential growth cone guidance intermediary in the context of spinal lateral motor column (LMC) motor axon trajectory selection in the limb mesenchyme. Using in situ mRNA detection, we first show paxillin expression in LMC neurons of chick and mouse embryos at the time of spinal motor axon extension into the limb. Paxillin loss-offunction and gain-of-function using in ovo electroporation in chick LMC neurons, of either sex, perturbed LMC axon trajectory selection, demonstrating an essential role of paxillin in motor axon guidance. In addition, a neuron-specific paxillin deletion in mice led to LMC axon trajectory selection errors. We also show that knocking down paxillin attenuates the growth preference of LMC neurites against ephrins in vitro, and erythropoietin-producing human hepatocellular (Eph)-mediated retargeting of LMC axons in vivo, suggesting paxillin involvement in Eph-mediated LMC motor axon guidance. Finally, both paxillin knockdown and ectopic expression of a nonphosphorylable paxillin mutant attenuated the retargeting of LMC axons caused by Src overexpression, implicating paxillin as a Src target in Eph signal relay in this context. In summary, our findings demonstrate that paxillin is required for motor axon guidance and suggest its essential role in the ephrin-Eph signaling pathway resulting in motor axon trajectory selection.

Key words: axon guidance; motor neuron; paxillin; spinal cord

\section{Significance Statement}

During the development of neural circuits, precise connections need to be established among neurons or between neurons and their muscle targets. A protein family found in neurons, Eph, is essential at different stages of neural circuit formation, including nerve outgrowth and pathfinding, and is proposed to mediate the onset and progression of several neurodegenerative diseases, such as Alzheimer's disease. To investigate how Ephs relay their signals to mediate nerve growth, we investigated the function of a molecule called paxillin and found it important for the development of spinal nerve growth toward their muscle targets, suggesting its role as an effector of Eph signals. Our work could thus provide new information on how neuromuscular connectivity is properly established during embryonic development.

Received Nov. 11, 2020; revised Mar. 4, 2021; accepted Mar. 7, 2021.

Author contributions: C.-J.C. and T.-J.K. designed research; W.-L.T., M.-Y.C., Y.-H.W., P.-S.C., K.-L.L., and A.K. performed research; W.-L.T., C.-Y.W., T.-I.H., M.-Y.C., J.-Y.C., and A.K. contributed unpublished reagents/ analytic tools; W.-L.T., C.-J.C., C.-Y.W., T.-I.H., Y.-H.W., J.-Y.C., A.K., and T.-J.K. analyzed data; T.-J.K. wrote the paper.

This work was supported by the Ministry of Science and Technology, Taiwan (MOST Grant 109-2320-B038-015, Grant 110-2636-B-038-004), the Cathay General Hospital (Grant 105CGH-TMU-02), and the TMU Research Center of Cancer Translational Medicine from the Featured Areas Research Center Program within the framework of the Higher Education Sprout Project by the Ministry of Education in Taiwan. We thank Charlene Chao for secretarial assistance.

${ }^{*}$ W.-L.T. and C.-J.C. contributed equally to this work.

The authors declare no competing financial interests.

Correspondence should be addressed to Tzu-Jen Kao at geokao@tmu.edu.tw.

https://doi.org/10.1523/JNEUROSCI.2863-20.2021

Copyright $\odot 2021$ the authors 


\section{Introduction}

During neural functional circuit assembly, neuronal axonal growth cones must be precisely guided to their proper targets, which is mediated by the activation of cell surface guidance receptors at the periphery; but, how receptor signals are transmitted to the cytoskeleton at the growth cone level is still unclear. Paxillin has been proposed to mediate cytoskeletal reorganization. Thus, our aim was to determine its function in the guidance of spinal motor axons into the limb mesenchyme.

Paxillin is a member of focal adhesion kinases, first identified as a scaffold protein with a primary role in cell adhesion (Turner et al., 1990). In addition, paxillin has been suggested to mediate several cellular functions essential for embryo development, including cell migration and survival (Turner, 1991; Turner et al., 1993; Price et al., 1998; Chay et al., 2002; Brown and Turner, 2004; Melendez et al., 2004; Sorenson, 2004; Subauste et al., 2004; Sheibani et al., 2008). In the nervous system, paxillin has been proposed to mediate key physiological events, including neuronal and Schwann cell migration and neurite formation (Leventhal and Feldman, 1996; Vindis et al., 2004; Guan et al., 2007; Miyamoto et al., 2012; Rashid et al., 2017). So far, despite the potential role of paxillin as an intermediary downstream of ephrin-Eph (erythropoietin-producing human hepatocellular) signaling, which mediates axon growth and pathfinding via interactions with Src family kinases, the role of paxillin in axon guidance remains unclear (Schaller and Parsons, 1995; Cowan and Henkemeyer, 2001; Carter et al., 2002; Baldwin et al., 2006). The essential functions of paxillin in axon guidance in vivo as well as the underlying mechanism of paxillin-mediated axon guidance events, including (1) its role as a signaling intermediary downstream of EphA versus EphB tyrosine kinase receptors, and (2) its involvement in other signaling systems, remain to be clarified.

A simple neuronal circuit suited for the study of axon guidance signaling is formed by the axon trajectories of spinal motor neurons innervating the limb muscles and resides in the lateral motor column (LMC) of the spinal cord (Tosney and Landmesser, 1985). LMC neurons are myotopically organized as follows: the lateral divisions of LMC neurons invariantly innervate the dorsal limb muscles, while the medial divisions of LMC neurons innervate the ventral ones (Landmesser, 1978; Lance-Jones and Landmesser, 1981; Luria and Laufer, 2007). Lateral and medial LMC motor neurons express the LIM homeodomain transcription factors Lim1 and Isl1, which control LMC axonal trajectories, in part, by restricting the expression of EphA4 and EphB1 receptors to lateral and medial LMC axons, respectively. Their ligands, ephrins, are expressed in the ventral (ephrin-As) or dorsal (ephrin-Bs) limb mesenchyme, and repulsive ephrin-Eph signaling has been proposed to mediate the proper selection of limb nerve trajectories (Tsuchida et al., 1994; Helmbacher et al., 2000; Eberhart et al., 2002; Kania and Jessell, 2003; Luria et al., 2008). In addition to Eph signaling, our evidence suggests that Netrin-1, expressed in the limb, and its receptors Unc5c and Dcc found in both subtypes of LMC neurons, also contribute to axon guidance (Poliak et al., 2015). This simple binary target choice of LMC axons is thus an ideal in vivo system to study the molecular cascade downstream of axon guidance receptors.

Here, we first show that paxillin is expressed in LMC neurons during motor axon outgrowth in the limb. Paxillin loss-of-function and gain-of-function perturb LMC axon trajectory selection with opposite effects. We also show that paxillin knockdown attenuates the growth preference of LMC neurites against ephrins, but not Netrin-1 in vitro and Eph receptor- and Srcmediated redirections of LMC axons in vivo. Combined, these results demonstrate that paxillin functions as an essential signaling intermediary downstream of Src in ephrin-Eph-mediated LMC axon trajectory selection.

\section{Materials and Methods}

Animals. Fertilized chick eggs (JD-SPF Biotech) were stored for a maximum of 1 week at $18^{3} \mathrm{C}$, incubated at $38^{3} \mathrm{C}$, and staged according to standard protocols (Hamburger and Hamilton, 1951). Mice were housed in an air-conditioned vivarium with food and water available ad libitum and with a $12 \mathrm{~h}$ light/dark cycle. All protocols of animal handling, including for chick and mouse embryos, were approved by the Institutional Animal Care and Use Committee of Taipei Medical University (Taipei, Taiwan).

Molecular biology. The characterization of expression constructs, including e[Isl1]::GFP, EphA4::GFP, EphB2::GFP, paxillin, and Src, has been previously described (Petit et al., 2000; Kania and Jessell, 2003; Knöll and Drescher, 2004; Kao et al., 2009).

In situ hybridization cDNA probes were prepared as described previously (Kao et al., 2009). In brief, target sequence amplification primers were designed using Primer3 version 0.4.0 software (Rozen and Skaletsky, 2000), and the probe size was set in the range of 600-800 bp. One-step RT-PCR was performed (Qiagen) using the designed primers containing T7 polymerase promoters (Thermo Fisher Scientific) to obtain a cDNA template from chick Hamburger-Hamilton stage ( $\mathrm{HH}$ st.) $25 / 26$, or mouse embryonic day 11.5 (E11.5) pooled brain RNA. The PCR product was purified by gel electrophoresis in $1 \%$ agarose gel and extracted using QIAquick Gel Extraction Kit (Qiagen). The purified DNA was then reamplified by PCR. The DNA yield was estimated by the Low DNA Mass Ladder (Thermo Fisher Scientific) after gel electrophoresis. DIG-labeled RNA probes were synthesized by in vitro transcription with T7 RNA polymerase using DIG RNA Labeling Kit (Roche). All probes were verified by sequencing. The sequence source and the recognized region for each probe are described as follows: chick paxillin (NM_204984, 400-1132); and mouse paxillin (NM_011223, 2117-2728). Details on Isl1 and Lim1 probes were described previously (Tsuchida et al., 1994).

Chick in ovo electroporation. Spinal cord electroporation of expression plasmids was performed at $\mathrm{HH}$ st. 18/19, in chicks of either sex, before the growth of LMC axons out of the spinal cord, as described previously (Momose et al., 1999; Luria et al., 2008; Kao et al., 2009). In brief, a 5-8 $\mu \mathrm{g} / \mu \mathrm{l}$ solution of plasmid DNA in TE buffer, $\mathrm{pH} 7.5$ (10 mм Tris$\mathrm{HCl}$ and $1 \mathrm{~mm}$ EDTA; Thermo Fisher Scientific), was injected into the lumbar neural tube through a small eggshell window under a stereomicroscope (SAGE Vision). The lower bodies of chick embryos were then electroporated with platinum/iridium electrodes (FHC) and the TSS20 Ovodyne electroporator (settings: $30 \mathrm{~V}, 5$ pulses, $50 \mathrm{~ms}$ wide in a $1 \mathrm{~s}$ interval; Intracel). Shell windows were sealed with Parafilm (Pechinery Plastic Packaging) and incubated at $38^{3} \mathrm{C}$. LMC axons normally reach the base of limbs at HH st. 23/24 (E4) and start to make a dorsoventral choice at HH st. 25/26 (E5; Hamburger and Hamilton, 1951). Chick embryos were thus harvested at HH st. 28/29 (E6) to analyze both outgrowth and proper dorsoventral choice of LMC axons. The efficiency of electroporation varied in the range of $5-30 \%$ of total LMC neurons electroporated depending on construct size and DNA concentration. When untagged expression plasmids were electroporated together with GFP expression plasmids or other plasmids fused with GFP, their concentration was at least three times that of GFP-fused plasmids to ensure a high coexpression efficiency. siRNA duplex oligonucleotides with a $3^{\prime} \mathrm{TT}$ overhang were purified over MicroSpin G-25 Columns (GE Healthcare) in TE buffer with $20 \mathrm{~mm} \mathrm{NaCl}$ (Sigma-Aldrich). A GFP expression plasmid $(1 \mu \mathrm{g} / \mu \mathrm{l})$ was coelectroporated with the siRNA solution to label motor axons. siRNA sequences (sense strand) were as follows: [paxillin] siRNA, 1:1 mixture of GCAGAACCATCTCCCACAA and GCGTCCCT CTCTGACTTTA; and scrambled [paxillin]siRNA, 1:1 mixture of GCACCACTACCTACGACAA and GCGTCCCTCAGTTCTCTTA. 
Horseradish peroxidase retrograde labeling of LMC motor neurons. Retrograde labeling of chick and mouse motor neurons was performed as described previously (Luria et al., 2008; Poliak et al., 2015). In brief, chick HH st. 28/29 embryos were harvested and incubated in Tyrode's solution ( $139 \mathrm{~mm} \mathrm{NaCl}, 3 \mathrm{~mm} \mathrm{KCl}, 17 \mathrm{~mm} \mathrm{NaHCO}_{3}, 12 \mathrm{~mm}$ glucose, 12 $\mathrm{mm} \mathrm{CaCl} 2,1 \mathrm{~mm} \mathrm{MgCl}_{2}$; Thermo Fisher Scientific) at room temperature (RT) and aerated with $95 \%$ air and $5 \% \mathrm{CO}_{2}$. The retrograde tracer used was a $20 \%$ solution of horseradish peroxidase (HRP; Roche) made by dissolving $100 \mathrm{mg}$ of $\mathrm{HRP}$ in $450 \mu \mathrm{l}$ of PBS $(137 \mathrm{~mm} \mathrm{NaCl}, 2.7 \mathrm{~mm} \mathrm{KCl}$, $4.3 \mathrm{mM} \mathrm{Na}_{2} \mathrm{HPO}_{4}, 1.47 \mathrm{~mm} \mathrm{KH}_{2} \mathrm{PO}_{4}$ ) with $50 \mu \mathrm{l}$ of $10 \%$ lysophosphatidylcholine (Fluka) in PBS. The HRP solution was injected into either dorsal or ventral hindlimb shank musculature of chick embryos. For mouse retrograde labeling, E12.5 embryos of either sex were dissected at the thoracic level and incubated in aerated DMEM/F12 medium (Thermo Fisher Scientific) at RT. The retrograde tracer used was a $20 \%$ HRP solution (Roche) made by dissolving $100 \mathrm{mg}$ of HRP in $450 \mu \mathrm{l}$ of PBS with $50 \mu$ of $10 \%$ lysophosphatidylcholine (Fluka) in PBS. The HRP solution was injected into either the dorsal or ventral forelimb proximal muscle group, and the embryos were incubated at $32^{3} \mathrm{C}$ under an infrared lamp and aerated with $95 \%$ air and $5 \% \mathrm{CO}_{2}$. Fresh medium was added every $30 \mathrm{~min}$ for $5 \mathrm{~h}$.

In vitro stripe assay. Protein carpets were prepared using silicon matrices with a channel system as described previously (Knöll et al., 2007). Carpets contained an alternating stripe pattern and were deposited in the following order: the first stripe contained a mixture of ephrin-Fc (or Netrin-1) and Fc-specific conjugated Cy3 (4:1 weight ratio), while the second stripe contained only Fc reagents without Fc-specific conjugated Cy3. E5 chick spinal motor columns were dissected and collected as described previously (Gallarda et al., 2008; Kao and Kania, 2011). In brief, E5 chick embryos of either sex were collected in ice-cold motor neuron medium [Neurobasal (Thermo Fisher Scientific), B-27 supplement (1:50, Thermo Fisher Scientific), $0.5 \mathrm{~mm}$ L-glutamate (SigmaAldrich), $25 \mathrm{~mm}$ L-glutamine (Thermo Fisher Scientific), and PenicillinStreptomycin (1:100; Wisent)]. The lumbar spinal cord was opened at the dorsal midline to allow the excision of motor columns under a stereomicroscope. Spinal motor columns were recognizable as the bulging part of the open-book spinal cord. Sharp tungsten needles (World Precision Instruments) were used to remove the dorsal spinal cord at the lateral part and the floor plate and medial motor column at the middle part of the open-book spinal cord. The excised motor column was then trimmed into square-shaped explants with an approximately one-quarter width of the motor column. Ten to 20 explants were then plated on laminin $(20 \mu \mathrm{g} / \mathrm{ml}$; Thermo Fisher Scientific) precoated $60 \mathrm{~mm}$ tissue culture dishes (Sarstedt) containing different combinations of stripe carpets in motor neuron medium and incubated in $95 \%$ air and $5 \% \mathrm{CO}_{2}$ atmosphere at $37^{3} \mathrm{C}$ for $18 \mathrm{~h}$.

\section{In situ mRNA detection and immunostaining.}

Chick and mouse embryos were fixed in a $4 \%$ solution of paraformaldehyde (Sigma-Aldrich) in PBS, equilibrated with $30 \%$ sucrose in PBS, embedded in O.C.T. compound (Sakura Finetek), and stored at $-80^{3} \mathrm{C}$. Twelve micrometer sections were collected using a cryostat microtome (Leica).

In situ mRNA detection was performed as described previously (Schaeren-Wiemers and Gerfin-Moser, 1993; Kania and Jessell, 2003). In brief, tissue sections were first fixed in $4 \%$ paraformaldehyde in PBS for $10 \mathrm{~min}$ at RT, washed three times with PBS, and digested with Proteinase K solution [ $1 \mu \mathrm{g} / \mathrm{ml}$ Proteinase K (Roche) in $6.25 \mathrm{~mm}$ EDTA, pH 8.0, and 50 mм Tris, pH 7.5 (Thermo Fisher Scientific)]. Samples were acetylated for $10 \mathrm{~min}$ by immersion in a $6 \mathrm{ml}$ mixture of triethanolamine (Sigma-Aldrich), $500 \mathrm{ml}$ of double-distilled $\mathrm{H}_{2} \mathrm{O}$, and $1.30 \mathrm{ml}$ of acetic anhydride (Sigma-Aldrich). After washing with PBS, samples were incubated with hybridization buffer [ $50 \%$ formamide, $5 \times$ SSC $(0.75 \mathrm{M}$ $\mathrm{NaCl}, 0.075 \mathrm{M} \mathrm{NaAc}$ ), $5 \times$ Denhardt's solution (Sigma-Aldrich), and 500 $\mu \mathrm{g} / \mathrm{ml}$ salmon sperm DNA (Roche)] for $2 \mathrm{~h}$ at RT followed by incubation overnight at $72^{3} \mathrm{C}$ with DIG-labeled RNA probes (see above) in hybridization buffer at a concentration of $2-5 \mathrm{ng} / \mu$ l. After hybridization, samples were immersed in $5 \times \mathrm{SSC}$ at $72^{3} \mathrm{C}$, followed by two washes in $0.2 \times$ SSC at $72^{3} \mathrm{C}$ for $45 \mathrm{~min}$ each and $0.2 \times$ SSC at RT for $5 \mathrm{~min}$. Tissues
Table 1. Antibodies and Fc reagents used

\begin{tabular}{|c|c|c|c|}
\hline $\begin{array}{l}\text { Antigen/pure } \\
\text { protein }\end{array}$ & Source species & Dilution & Source/reference \\
\hline EphA4 & Rabbit & $1: 500$ & $\begin{array}{l}\text { Santa Cruz } \\
\text { Biotechnology }\end{array}$ \\
\hline EphB1 & Goat & $1: 500$ & $\begin{array}{l}\text { Santa Cruz } \\
\text { Biotechnology }\end{array}$ \\
\hline Paxillin & Rabbit & $1: 500$ & Abcam \\
\hline Paxillin & Mouse & $1: 500$ & Thermo Fisher Scientific \\
\hline \multirow[t]{2}{*}{ Neurofilament } & Mouse $(2 \mathrm{H} 3)$ & $1: 100$ & DSHB \\
\hline & Mouse (3A10) & 1:100 & DSHB \\
\hline$|s| 1 / 2$ & Mouse & $1: 100$ & DSHB \\
\hline $\operatorname{Lim} 1 / 2$ & Mouse & $1: 100$ & DSHB \\
\hline HRP & Goat & $1: 2000$ & Roche \\
\hline Foxp1 & Rabbit & $1: 1000$ & Abcam \\
\hline GFP & Guinea pig & $1: 1000$ & AbD Serotec \\
\hline GFP & Rabbit & $1 / 1000$ & Molecular Probes \\
\hline Ephrin-A2-Fc & Human & $10 \mu \mathrm{g} / \mathrm{ml}$ & R\&D Systems \\
\hline Ephrin-A5-Fc & Human & $10 \mu \mathrm{g} / \mathrm{ml}$ & R\&D Systems \\
\hline Ephrin-B1-Fc & Mouse & $10 \mu \mathrm{g} / \mathrm{ml}$ & R\&D Systems \\
\hline Ephrin-B2-Fc & Mouse & $10 \mu \mathrm{g} / \mathrm{ml}$ & R\&D Systems \\
\hline EphA2-Fc & Mouse & $10 \mu \mathrm{g} / \mathrm{ml}$ & R\&D Systems \\
\hline EphB1-Fc & Rat & $10 \mu \mathrm{g} / \mathrm{ml}$ & R\&D Systems \\
\hline Netrin-1 & Mouse & $250 \mathrm{ng} / \mathrm{ml}$ & R\&D Systems \\
\hline $\mathrm{Fc}$ & Human & $10 \mu \mathrm{g} / \mathrm{ml}$ & R\&D Systems \\
\hline Anti-Fc & Mouse & $\begin{array}{l}\text { 1:4 mass ratio to } \\
\text { ephrin }\end{array}$ & Sigma-Aldrich \\
\hline Goat & $\begin{array}{l}\text { 1:4 mass ratio to } \\
\text { ephrin }\end{array}$ & Sigma-Aldrich & \\
\hline
\end{tabular}

DSHB, Developmental Studies Hybridoma Bank.

were then rinsed with $\mathrm{B} 1$ buffer $(0.1 \mathrm{M}$ Tris, $\mathrm{pH} 7.5$, and $0.15 \mathrm{M} \mathrm{NaCl})$ for 5 min, blocked with B2 buffer (10\% heat-inactivated horse serum in B1) for $1 \mathrm{~h}$ at RT, and incubated with anti-DIG antibody (1:5000 in B2; Roche) overnight at $4^{3} \mathrm{C}$. Samples were then rinsed with $\mathrm{B} 1$ and equilibrated with B3 buffer ( $0.1 \mathrm{~m}$ Tris, pH 9.5, $\left.0.1 \mathrm{M} \mathrm{NaCl}, 0.05 \mathrm{M} \mathrm{MgCl}_{2}\right)$. To detect bound anti-DIG antibodies, samples were incubated with B4 buffer [100 mg/ml NBT, $50 \mathrm{mg} / \mathrm{ml} \mathrm{BCIP} \mathrm{(Roche),} \mathrm{and} 400 \mathrm{~mm}$ levamisole (Sigma-Aldrich) in B3] in the dark. The reaction was stopped by immersion in $\mathrm{H}_{2} \mathrm{O}$.

Immunostaining was performed as described previously (Kao et al., 2009; Kao and Kania, 2011). In brief, sectioned tissue or cultured explants were washed in PBS, incubated in blocking buffer [ $1 \%$ heatinactivated horse serum in $0.1 \%$ Triton X-100/PBS (Sigma-Aldrich)] for $5 \mathrm{~min}$, followed by incubation overnight at $4^{3} \mathrm{C}$ in selected primary antibodies diluted in blocking solution. After incubation in primary antibodies, samples were washed with PBS and incubated with the appropriate secondary antibodies for $1 \mathrm{~h}$ at RT. See Table 1 for the list of antibodies used.

Image quantification. Images were acquired using a Leica DM6000 Microscope or an EVOS FL Microscope (Thermo Fisher Scientific). GFP-labeled axonal projections were quantified by combining overthreshold pixel counts in limb section images containing limb nerves (10-15 limb sections with $12 \mu \mathrm{m}$ thickness each) using Photoshop (Adobe). The dorsal or ventral limb nerve was selected by gating the neurofilament channel and using the Lasso Tool, and pixel counts from the threshold to the maximal level were indicated in the histogram window of the GFP channel. Motor neuron numbers were quantified by combining the cell counts of a series of spinal cord section images (5-10 limb sections from each embryo) using Photoshop. LMC axon outgrowth was quantified by comparing the ratio of average lengths of GFP-labeled LMC axons between each experimental group and the GFP control. For retrograde tracing experiments, proportions of medial or lateral LMC axon trajectory selection were quantified by combining cell counts of tracer-filled, electroporated medial $\left(\mathrm{HRP}^{+}, \mathrm{GFP}^{+}, \mathrm{Isll}^{+}\right)$and lateral $\left(\mathrm{HRP}^{+}, \mathrm{GFP}^{+}, \mathrm{Isll}^{-}\right)$LMC neurons for dorsally filled embryos, or tracer-filled, electroporated lateral $\left(\mathrm{HRP}^{+}, \mathrm{GFP}^{+}, \mathrm{Liml}^{+}\right)$and medial $\left(\mathrm{HRP}^{+}, \mathrm{GFP}^{+}, \mathrm{Lim}^{-}\right) \mathrm{LMC}$ neurons for ventrally filled embryos. 


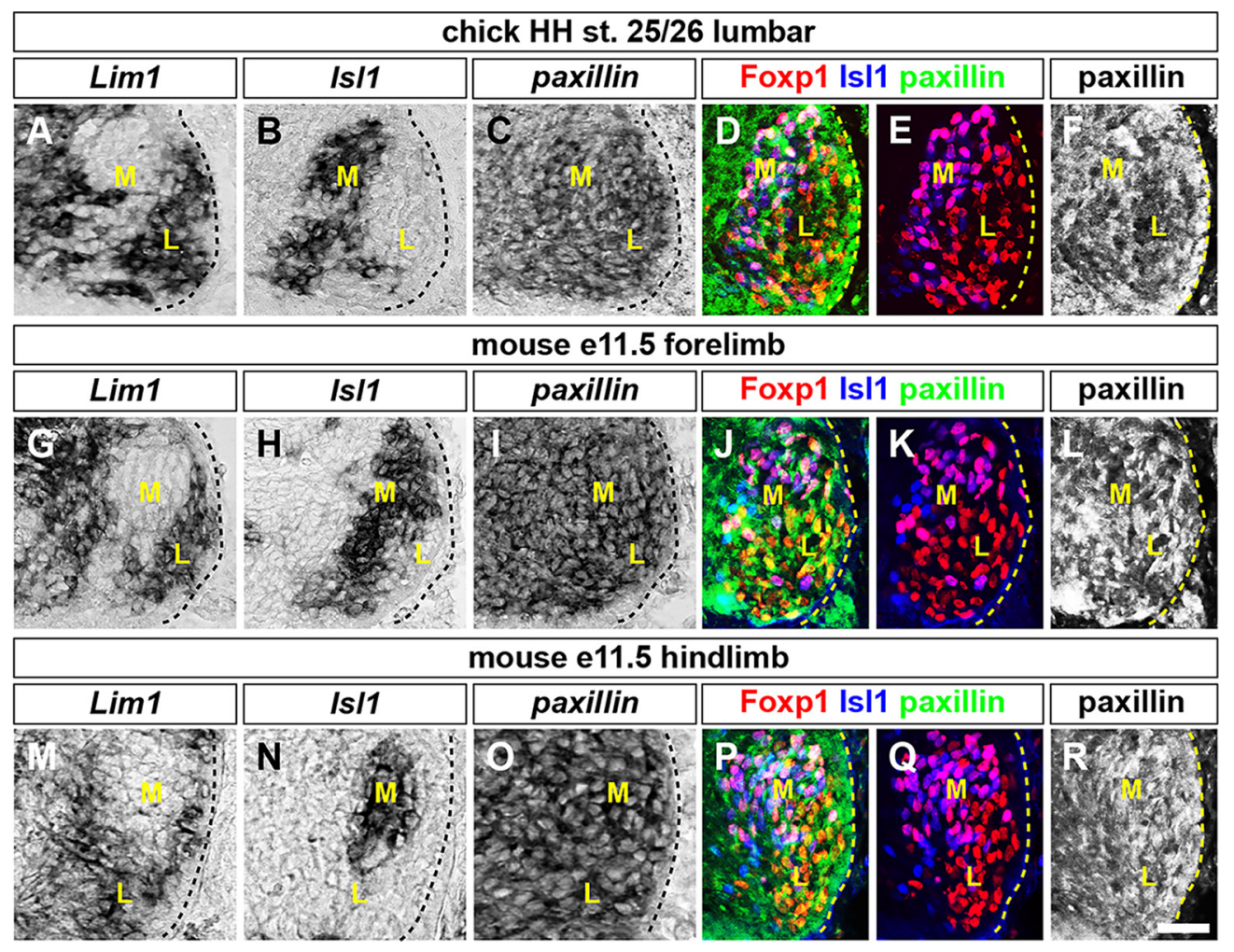

Figure 1. Expression of paxillin in chick and mouse LMC motor neurons. All chick sections are HH st. $25 / 26$ lumbar spinal cord; all mouse sections are E11.5 lumbar spinal cord. $\boldsymbol{A}-\boldsymbol{C}$, G-I, $\mathbf{M - 0}$, Detection of mRNA in consecutive sections of spinal cords. $\boldsymbol{A}, \boldsymbol{B}$, Detection of $\operatorname{Lim} 1(\boldsymbol{A})$ and $I s / 1(\boldsymbol{B}) \mathrm{mRNA}$ in the chick spinal cord, highlighting lateral and medial LMC neurons, respectively. C, Detection of paxillin mRNA in both medial and lateral LMC neurons. $\mathbf{G}, \boldsymbol{H}, \boldsymbol{M}, \mathbf{N}$, Detection of $\operatorname{Lim} 1(\mathbf{G}, \boldsymbol{M})$ and $I s / 1(\boldsymbol{H}, \boldsymbol{N})$ mRNA in mouse $L M C$ neurons. I, $\mathbf{0}$, Detection of paxillin mRNA in both medial and lateral mouse LMC neurons. $\boldsymbol{D}-\boldsymbol{F}, \boldsymbol{J}-\boldsymbol{L}, \boldsymbol{P}-\boldsymbol{R}$, Detection of protein in sections of spinal cords. $\boldsymbol{D}, \boldsymbol{E}$, Detection of Foxp1 and Isl1 in the chick spinal cord, highlighting lat-

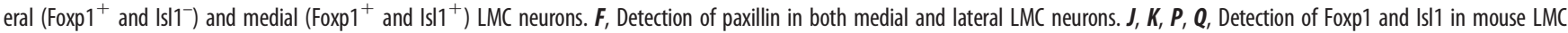
neurons. $\boldsymbol{L}, \boldsymbol{R}$, Detection of paxillin in both medial and lateral mouse LMC neurons. M, Medial; $L$, lateral. Scale bar, (in $\boldsymbol{R}) \boldsymbol{A}-\boldsymbol{R}, 30 \mu \mathrm{m}$.

The proportion of GFP- or EphA4-labeled neurites of cultured motor neuron explants growing on each stripe type was quantified by combining threshold pixel quantification over either the first or second stripe type in multiple images using Photoshop. The ratio of immunoreactivity signal levels of paxillin mRNA in LMC neurons of the electroporated to the unelectroporated contralateral side (e/u ratio) were quantified from the combined pixel intensities of paxillin mRNA signals in multiple images using Photoshop, and neighboring sections labeled with Foxp1 and Isl1 were used as references to determine the area of LMC.

To minimize possible experimental bias, multiple limb sections were selected every two to three sections anteroposteriorly until the entire crural plexus of limb nerves in each embryo was covered, while cultured LMC explants were randomly selected for quantification. In addition, the proportion of total and electroporated motor neuron cells was quantified for most experimental groups to ensure cell identity and exclude abnormal cell death before the subsequent analysis of axon growth preferences (dorsal/ventral, medial/lateral, or first stripe/s stripe).

Statistical analysis. Data from experimental replicate sets were evaluated using Microsoft Excel. The combined proportions or cell numbers were compared using the Fisher's exact test for the proportion of medial/lateral LMC neurons in retrograde labeling experiments, and the Mann-Whitney $U$ test for the dorsal/ventral preference of limb nerves or cultured LMC neurites growing on the first or second stripe with the threshold for significance set at 0.05 (Poliak et al., 2015).

\section{Results}

Paxillin expression in LMC motor neurons

Paxillin has been proposed to mediate various cellular events related to cell adhesion and cytoskeletal reorganization, including neuronal migration and axon outgrowth (Deakin and
Turner, 2008). To investigate paxillin function in motor axon guidance in vivo and to define its role, we first determined whether paxillin is expressed in LMC when motor axons grow into the limb mesenchyme, between $\mathrm{HH}$ st. 25 and 27 in chick embryos and between E10.5 and E11.5 in mouse embryos (Hamburger and Hamilton, 1951; Tosney and Landmesser, 1985; Kania et al., 2000). We thus checked paxillin expression in the lumbar spinal cord of $\mathrm{HH}$ st. 25/26 chick embryos and both brachial and lumbar spinal cords of E11.5 mouse embryos. Subpopulations of LMC neurons innervating the dorsal and ventral limb muscles were identified by the expression of the lateral LMC marker Lim 1 or the medial LMC neuron marker Isl1 (Fig. $1 A, B, G, H, M, N)$. The paxillin mRNA was found to be highly expressed in both lateral and medial LMC neurons in chick and mouse embryos (Fig. 1C,I,O). We also detected the expression of paxillin protein in LMC neurons (Fig. $1 D-F, J-L, P-R$ ). No obvious difference in paxillin expression levels was observed between the medial and lateral LMC in chick and mouse or between the brachial and lumbar spinal cords of mouse embryos.

\section{Paxillin is required for limb trajectory selection by LMC} axons

To test whether paxillin is involved in the selection of dorsal or ventral limb nerves by LMC axons, we knocked down paxillin expression by introducing an inhibitory siRNA against paxillin mRNA ([paxillin]siRNA) into LMC neurons. To this end, we coelectroporated siRNA with a GFP expression plasmid into the chick lumbar neural tube before LMC neuron specification and axon entry into the limb at $\mathrm{HH}$ st. $18 / 19$, and examined $\mathrm{GFP}^{+}$ 

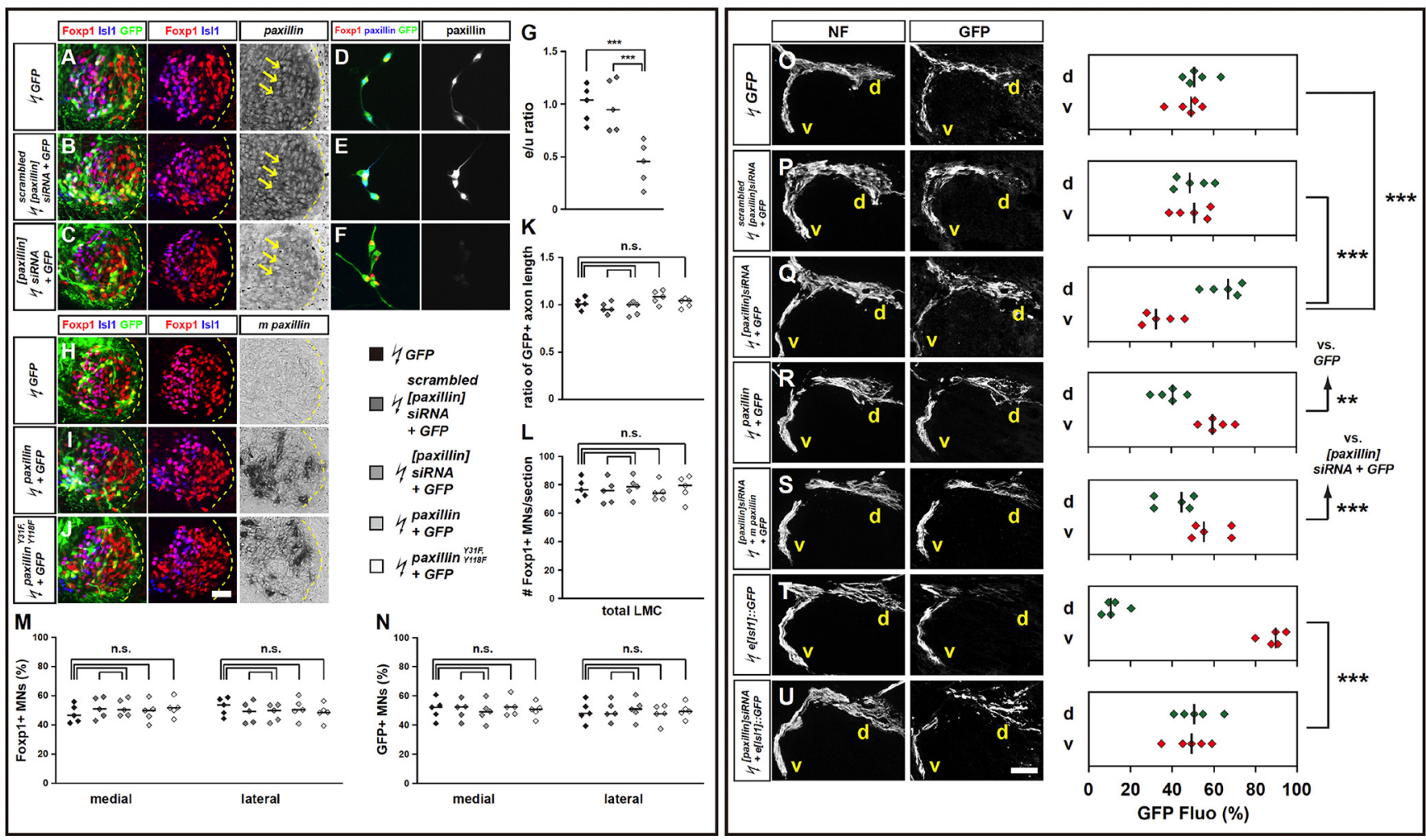

Figure 2. Paxillin function is required for the selection of limb axon trajectory. All images are from chick HH st. 28/29 lumbar levels. $A-C$, Detection of Foxp1, Isl1, GFP, and paxillin in LMC neurons of chick embryos electroporated with GFP $(\boldsymbol{A})$, scrambled [paxillin]siRNA and GFP $(\boldsymbol{B})$, or [paxillin]siRNA and GFP (C). D-F, Detection of Foxp1, paxillin, and GFP in dissociated LMC neurons purified from chick embryos electroporated with GFP (D), scrambled [paxillin]siRNA and GFP (E), or [paxillin]siRNA and GFP (F). G, Quantification of effects of GFP, scrambled [paxillin]siRNA, and GFP, or [paxillin]siRNA + GFP electroporation on paxillin mRNA levels. The e/u ratio was obtained in at least 15 sections of five embryos. $\boldsymbol{H}-\mathbf{J}$, Detection of Isl1, Foxp1, and GFP proteins and mouse paxillin mRNA in the LMC of chick HH st. 28/29 electroporated with GFP $(\boldsymbol{H})$, paxillin and GFP (I), or paxillin ${ }^{Y_{31 F}, \text { Y118F }}$ and GFP (I) expression plasmids. Mouse (m) paxillin probe designed to specifically recognize ectopic mouse paxillin mRNA following $m$ paxillin electroporation. $\boldsymbol{K}$, Quantification of effects of GFP, scrambled [paxillin]siRNA and GFP, [paxillin]siRNA and

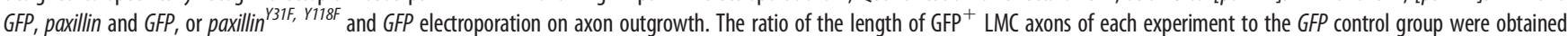
in at least 15 sections of five embryos. L, Number of LMC motor neurons expressed as the average number of total (Foxp $1^{+}$) LMC neurons per section (\# Foxp $1^{+}$MNs/section). Numbers of embryos: $n=5$ for all groups. $\boldsymbol{M}, \boldsymbol{N}$, Number of total or electroporated medial (Foxp $1^{+}|\mathrm{s}| 1^{+}$) and lateral (Foxp $\left.1^{+}|\mathrm{s}| 1^{-}\right) \operatorname{LMC}$ motor neurons in lumbar spinal cord expressed as the percentage of total motor neurons [Foxp1 $1^{+}$MNs (\%); $\boldsymbol{M}$ ] or electroporated motor neurons [GFP ${ }^{+}$MNs (\%); $\boldsymbol{N}$ ]. Numbers of embryos: $n=5$ for all groups. $\mathbf{0}-\boldsymbol{U}$, Neurofilament and GFP detection in the limb nerve branches in the crural plexus of chick embryos electroporated with the following expression plasmids and siRNAs: GFP (0), scrambled [paxillin]siRNA and GFP (P), [paxillin]siRNA and GFP (Q), paxillin and GFP (R), [paxillin]siRNA, mouse paxillin and GFP (S), medial LMC axonal marker, e[Is/1]]:GFP ( $\boldsymbol{T})$, or [paxillin]siRNA and e[Is/1]::GFP (U). Quantification of GFP signals in all electroporation experiments expressed as, respectively, percentage in dorsal and ventral limb nerves [GFP Fluo (\%)]. Numbers of embryos: $n=5$ for all groups. $d$, Dorsal; $v$, ventral. $n$.s. $=$ not significant; ${ }^{* * *} p<0.001 ;{ }^{* *} p<0.01$; statistical significance computed using Fisher's exact test $(\boldsymbol{A}-\boldsymbol{N})$ or Mann-Whitney $\boldsymbol{U}$ test $(\mathbf{0}-\boldsymbol{U})$; solid lines in each scatterplot show the median difference. Scale bars: $\mathbf{A}-\boldsymbol{C}, \boldsymbol{H}-\boldsymbol{J}, 45 \mu \mathrm{m} ; \mathbf{D}-\boldsymbol{F}, 35 \mu \mathrm{m} ; \mathbf{O}-\boldsymbol{U}, 150 \mu \mathrm{m}$.

motor axons in the dorsal and ventral divisional nerve branches exiting the crural plexus at HH. 28/29 (Kania and Jessell, 2003). Coelectroporation of [paxillin]siRNA and GFP plasmid significantly reduced paxillin $\mathrm{mRNA}$ and protein expression compared with embryos electroporated with a control GFP plasmid or scrambled [paxillin]siRNA (Fig. $2 A-G ; p=3.61 * 10^{-4}$ vs GFP, $p=5.25^{\star} 10^{-4}$ vs scrambled [paxillin]siRNA), but it did not cause obvious changes in axon outgrowth (Fig. $2 K ; p=0.6625$ vs GFP, $p=0.5174$ vs scrambled [paxillin] siRNA for the ratio of $\mathrm{GFP}^{+}$ axon length), nor did it change the LMC neuron numbers expressing the marker Foxp1 and proportions of lateral LMC $\left(\right.$ Foxp $1^{+}$, Isl1 $^{-}$) versus medial LMC (Foxp $1^{+}, \mathrm{Isl1}^{+}$) neurons when compared with embryos electroporated with a control GFP plasmid or scrambled [paxillin]siRNA (Fig. $2 L, M ; p=0.7538$ vs GFP, $p=0.5110$ vs scrambled [paxillin]siRNA for total Foxp $1^{+}$ neuron numbers/section; $p=0.2156$ vs GFP, $p=0.5335$ vs scrambled [paxillin]siRNA for the proportions of lateral or medial LMC neurons). Similar numbers of electroporated neurons were also found in both LMC divisions when comparing [paxillin]siRNA and GFP coexpressing groups with controls (Fig. $2 N ; p=0.3021$ vs GFP, $p=0.4671$ vs scrambled [paxillin]siRNA).
To determine whether paxillin knockdown affects the choice of limb trajectory by LMC axons, we quantified the proportions of $\mathrm{GFP}^{+}$axons in the dorsal and ventral limb nerve branches by integrating the fluorescence intensities of a series of hindlimb section images in multiple embryos for each experimental condition (Kania and Jessell, 2003; Luria et al., 2008). For statistical analysis, we compared the proportions of dorsally (or ventrally) projected $\mathrm{GFP}^{+}$axons between different experimental conditions. In embryos coelectroporated with [paxillin]siRNA and GFP, a significantly higher proportion of $\mathrm{GFP}^{+}$axons was observed in the dorsal nerve branches when compared with both GFP or scrambled [paxillin] siRNA controls (Fig. 2O-Q; $p=3.53$ * $10^{-4}$ vs GFP, $p=1.77^{\star} 10^{-4}$ vs scrambled [paxillin] siRNA). The axon misrouting effect was rescued by mouse paxillin coexpression (Fig. 2Q,S; $p=5.72^{\star} 10^{-5}$ ). These findings indicate that paxillin knockdown leads to a significantly higher proportion of LMC motor axons entering the dorsal limb.

We then performed paxillin gain-of-function experiments by coelectroporating paxillin and GFP expression plasmids into LMC neurons and analyzed motor axon trajectories in the limb. Compared with GFP controls, the axon length, specification, and 


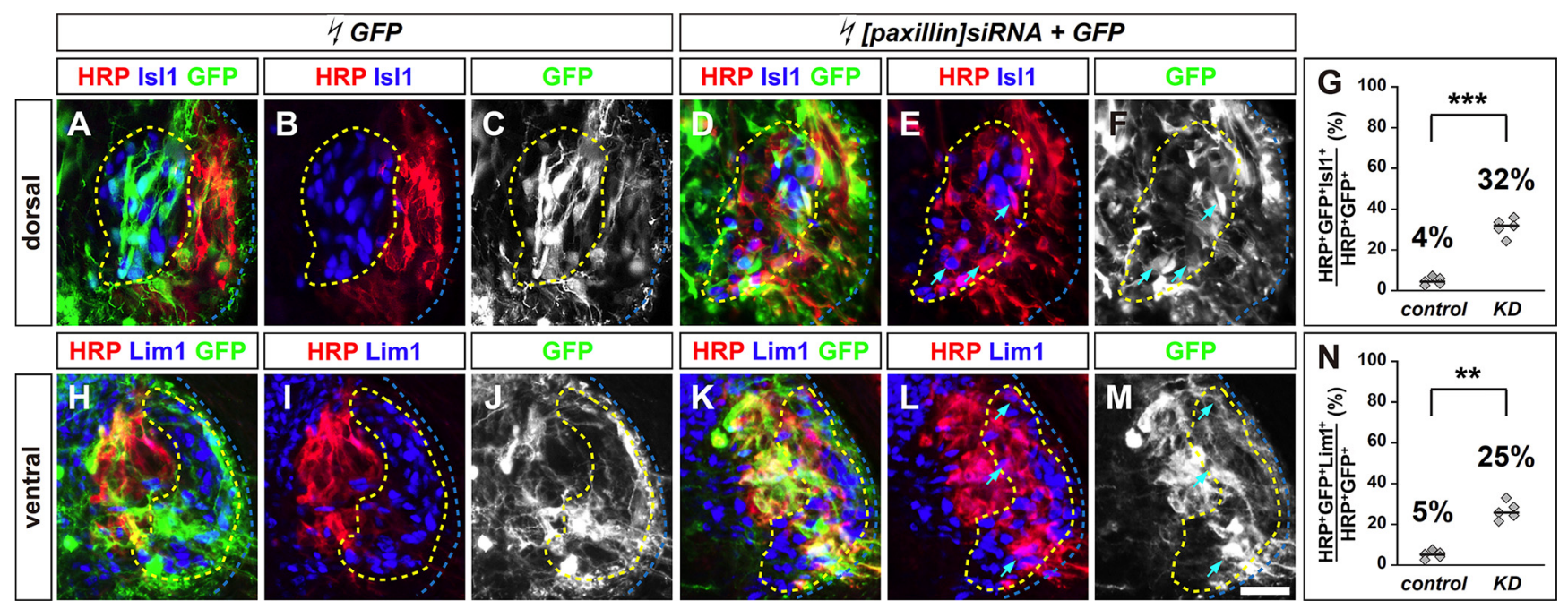

Figure 3. Paxillin function is required for the fidelity of LMC motor axon limb trajectories. Retrograde labeling of LMC neurons by HRP injections into the dorsal or ventral limb muscles of chick HH st. 28/29 embryos. $\boldsymbol{A}-\boldsymbol{F}$, Detection of HRP, Lim1, and GFP in the LMC regions of GFP (A-C) or of [paxillin]siRNA and GFP (D-F) electroporated embryos injected with HRP into dorsal hindlimb shank muscles. G, Proportions of electroporated medial LMC motor neurons labeled with HRP in dorsally filled embryos. Number of embryos: $\boldsymbol{n}=5$ for all groups. $\boldsymbol{H}-\boldsymbol{M}$, Detection of HRP, Lim1, and GFP in the LMC regions of GFP $(\boldsymbol{H}-\boldsymbol{J})$ or of [paxillin]siRNA and GFP $(\boldsymbol{K}-\boldsymbol{M})$ electroporated embryos injected with HRP into ventral hindlimb shank muscles. Examples of HRP ${ }^{+}$ $(\boldsymbol{L}) \mathrm{GFP}^{+}(M)$ neurons are indicated by arrows and arrowheads. $\boldsymbol{N}$, Proportions of electroporated lateral LMC motor neurons labeled with HRP in ventrally filled embryos. Number of embryos: $n=5$ for all groups. Control, GFP electroporated groups; KD, [paxillin]siRNA and GFP electroporated groups. ${ }^{* * *} p<0.001$; ${ }^{* *} p<0.01$; statistical significance was computed using Fisher's exact test; solid lines in each scatterplot show the median difference. Scale bars: $\mathbf{A}-\boldsymbol{F}, \boldsymbol{H}-\boldsymbol{M}, 45 \mu \mathrm{m}$.

survival of LMC neurons were normal in paxillin and GFP coexpressing embryos (Fig. $2 H, I, K-M ; p=0.2210$ for the ratio of $\mathrm{GFP}^{+}$axon length; $p=0.6616$ for total Foxp $1^{+}$neuron numbers/ section; $p=0.4559$ for the proportions of lateral or medial LMC neurons), and $\mathrm{GFP}^{+} \mathrm{LMC}$ neurons showed similar proportions of electroporated cells in both LMC divisions (Fig. $2 \mathrm{H}, I, N$; $p=0.5035)$. In paxillin and GFP coexpressing embryos, a significantly higher proportion of $\mathrm{GFP}^{+}$axons was observed in the ventral nerves when compared with GFP controls (Fig. 2O,R; $p=1.24^{*} 10^{-3}$ ), suggesting that paxillin overexpression leads to a significantly higher proportion of LMC motor axons entering the ventral limb. Together, these findings demonstrate that paxillin expression by LMC motor neurons is essential for the fidelity of LMC axon guidance in the limb.

The following two possible scenarios could explain the increased proportion of LMC neurons projecting into the dorsal nerve branch following paxillin knockdown: (1) some medial LMC axons enter the dorsal limb nerve; or (2) both medial and lateral LMC axons project into both limb nerves, but a greater proportion of medial LMC axons project into the dorsal limb nerve. In either case, we expected a loss of fidelity of medial LMC trajectory selection caused by paxillin knockdown. To determine whether paxillin knockdown results in the redirection of medial LMC axons into the dorsal limb mesenchyme, we coelectroporated [paxillin]siRNA with the e[Isl1]:: GFP plasmid, which preferentially labels medial LMC motor neurons and their axons (Kao et al., 2009), and as control, the e[Isl1]:: GFP plasmid only. In embryos coelectroporated with [paxillin] siRNA and the e[Isl1]::GFP, a significantly higher proportion of $\mathrm{GFP}^{+}$axons was observed in the dorsal limb nerve when compared with $e$ [Isl1]::GFP controls (Fig. 2T,U; $p=3.68 * 10^{-5}$ ). These findings indicate that paxillin is required for the fidelity of limb trajectory selection by medial LMC axons.

The $\mathrm{GFP}^{+}$axon-counting experiments do not allow us to compare the extent to which both medial and lateral divisions are sensitive to paxillin loss-of-function. To determine whether paxillin function is required for the selection of appropriate LMC axon trajectory, we labeled LMC neurons by HRP retrograde tracer injection into the dorsal or ventral shank muscles of $\mathrm{HH}$ st. 28/29 embryos electroporated with [paxillin]siRNA and GFP expression plasmids or GFP alone, and determined the LMC divisional identity of labeled neurons (Kania and Jessell, 2003; Kao et al., 2009). The proportion of electroporated medial LMC neurons labeled by dorsal limb HRP injections was significantly higher in paxillin knock-down embryos when compared with controls, indicating that paxillin is required for the choice of limb axon trajectory by medial LMC neurons (Fig. $3 A-G ; p=1.52{ }^{\star} 10^{-4}$ ). On the other hand, the proportion of lateral LMC labeled by ventral limb HRP injections was also significantly higher in paxillin knock-down embryos when compared with controls (Fig. $3 H-M ; p=0.0029$ ). These observations thus confirm that paxillin knockdown results in a loss of fidelity of limb trajectory choice by LMC axons, and that medial LMC axons rely on paxillin expression to a greater extent than lateral LMC axons.

\section{Asymmetric paxillin knock-down sensitivity of the LMC trajectory choice}

To ascertain whether paxillin function is required for the trajectory selection of motor axons from both LMC subpopulations, we next examined the LMC axon trajectory in paxillin mutant mice. Paxillin full deletion is lethal by E9.5, before the dorsoventral trajectory of LMC axons into the limb at E11.5 to E12.5 (Hagel et al., 2002). Therefore, we analyzed paxillin function in the LMC trajectory choice using mice with a neuron-specific paxillin deletion (Nes-Cre:Pxn ${ }^{F / F}$; Rashid et al., 2017). LMC neuron specification and survival were normal in Nes-Cre:Pxn ${ }^{F / F}$ mice compared with paxillin floxed $\left(P x n^{F / F}\right)$ only littermates (Fig. $4 A-I, p=0.3660$; Fig. $4 J, p=0.6844$ ). To trace the trajectory of LMC axons, we labeled LMC neurons by HRP retrograde tracer injection into the dorsal or ventral shank muscles of E12.5 Nes-Cre:Pxn ${ }^{F / F}$ and $P x n^{F / F}$ littermate embryos and quantified the proportions of tracer-filled LMC neurons expressing Isl1 and Lim1 (Kania et al., 2000; Chang et al., 2018). The proportion of medial LMC neurons labeled by dorsal limb HRP injection was significantly higher in Nes-Cre:Pxn ${ }^{F / F}$ embryos than in control 


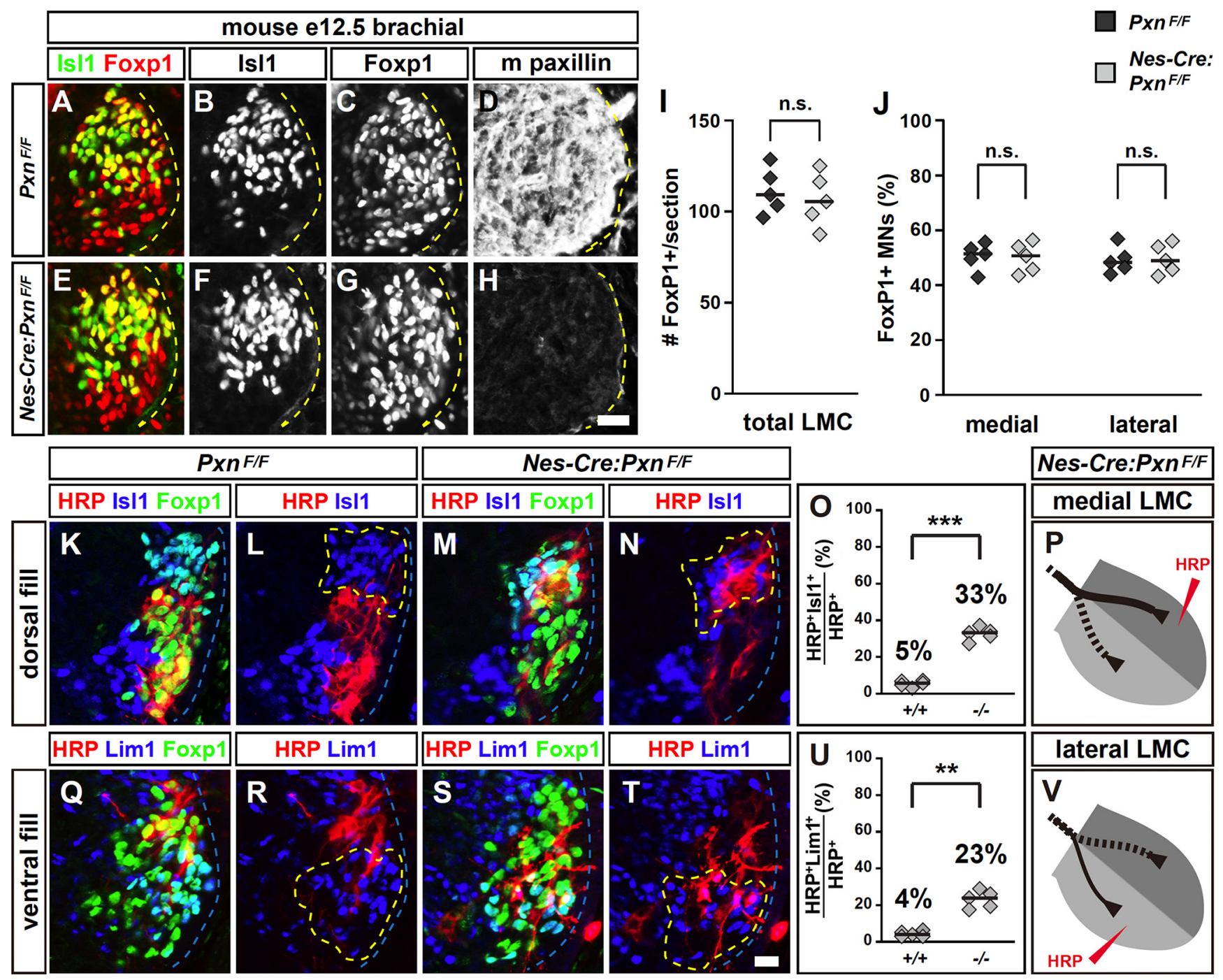

Figure 4. Paxillin is required for the fidelity of LMC motor axon trajectory selection. Retrograde labeling of LMC neurons by HRP injections into the dorsal or ventral limb muscles of mouse E12.5 embryos. $\boldsymbol{A}-\boldsymbol{H}$, Detection of Is1 (green), Foxp1 (red), and paxillin protein in the LMC region at the brachial level of mouse E12.5 Pxn ${ }^{F / F}(\boldsymbol{A}-\boldsymbol{D})$ or Nes-Cre:Pxn ${ }^{F / F}(\boldsymbol{E}-\boldsymbol{H})$ embryos. $\boldsymbol{I}$, Number of LMC motor neurons expressed as the average number of total (Foxp ${ }^{+}$) LMC neurons per section (\# FoxP1 $1^{+}$/section). Number of embryos: $n=5$ for all groups. J, Number of total medial (FoxP1 ${ }^{+}|\mathrm{s}| 1^{+}$) and lateral $\left(F o x P 1^{+}|s| 1^{-}\right)$LMC motor neurons in brachial spinal cord expressed as the percentage of total motor neurons [FoxP1 ${ }^{+}$MNs (\%)]. Number of embryos: $n=5$ for all groups. $\mathbf{K}-\mathbf{N}$, Detection of HRP (red), Is11 (blue), and Foxp1 (green), which marks all LMC neurons, in the LMC regions of $P x n^{F / F}(\boldsymbol{K}, \boldsymbol{L})$ and Nes-Cre:Pxn ${ }^{F / F}(\boldsymbol{M}, \boldsymbol{M})$ embryos injected with HRP into dorsal forelimb muscles. $\mathbf{0}$, Quantification of retrogradely labeled medial LMC axon projections. The graph depicts the percentage of $\mathrm{HRP}^{+}$motor neurons that express the medial LMC marker Isl1 following a dorsal limb injection. Numbers of embryos: $n=5$ for all groups. $\boldsymbol{P}$, Summary scheme of medial LMC projections in Nes-Cre:Pxn ${ }^{F / F}$ mice, depicting a significant misrouting of medial LMC axons into the dorsal limb. Q- $\boldsymbol{T}$, Detection of HRP (red), Lim1 (blue), and Foxp1 (green) in the LMC regions of $\operatorname{Pxn}^{\mathrm{F} / F}(\boldsymbol{Q}, \boldsymbol{R})$ and Nes-Cre:Pxn ${ }^{\mathrm{F} / F}(\boldsymbol{S}, \boldsymbol{I})$ embryos injected with HRP into ventral forelimb muscles. $\boldsymbol{U}$, Quantification of retrogradely labeled lateral LMC axon projections. The graph depicts the percentage of $\mathrm{HRP}^{+}$motor neurons that express the lateral LMC marker Lim1 following a ventral limb injection. Numbers of embryos: $n=5$ for all groups. $\boldsymbol{V}$, Summary scheme of lateral LMC projections in Nes-Cre:Pxn ${ }^{F / F}$ mice, depicting a significant misrouting of lateral LMC axons into the ventral limb. Pxn, Paxillin; Nes, nestin. n.s. = not significant; **** $<0.001$; ${ }^{* *} p<0.01$; statistical significance was computed using Fisher's exact test; solid lines in each scatterplot show the median difference. Scale bars: $\boldsymbol{H}$ (for $\left.\boldsymbol{A}-\boldsymbol{H}\right), \boldsymbol{T}($ for $\boldsymbol{K}-\boldsymbol{N}, \mathbf{Q}-\boldsymbol{T}), 20 \mu \mathrm{m}$.

embryos (Fig. $4 K-O ; p=5.52 * 10^{-5}$ ). The proportion of lateral LMC neurons labeled by ventral limb HRP injection was also significantly higher, but to a lesser extent, in Nes-Cre:Pxn ${ }^{F / F}$ embryos than in control embryos (Fig. $4 Q-U ; p=3.43{ }^{\star} 10^{-3}$ ). These observations demonstrate that paxillin function is required for the selection of appropriate LMC axon trajectories, and that there is a different dependence on paxillin function between medial and lateral LMC neurons (Fig. $4 P, V$ ).

Paxillin is specifically required for ephrin-mediated motor axon responses

Paxillin has been suggested to act as a downstream effector of Eph signaling in several contexts (Vindis et al., 2004; Baldwin et al., 2006). Additionally, ephrin-Eph signaling is sufficient to redirect LMC axons (Kania and Jessell, 2003; Luria et al., 2008). To investigate the dependence of Eph signaling on paxillin function, we tested the response of LMC axons to stripes of ephrin-A or ephrin-B proteins in the context of paxillin loss-of-function (Kao and Kania, 2011). HH st. 25/26 LMC explants were dissected and placed onto carpets of two alternating stripes: those containing a mixture of ephrin-Fc and a Cy3 secondary antibody and those containing Fc protein only. Medial LMC axons were identified by e[Isl1]::GFP electroporation, whereas lateral LMC axons were identified by their EphA4 expression. Stripe preference was scored as the proportion of GFP or EphA4 signal found over different stripes after overnight explant culture (Kao and Kania, 2011; Kao et al., 2015). For statistical analysis, we compare the proportions of neurite growth preference in ephrin (or Fc) stripes between different experimental conditions. 


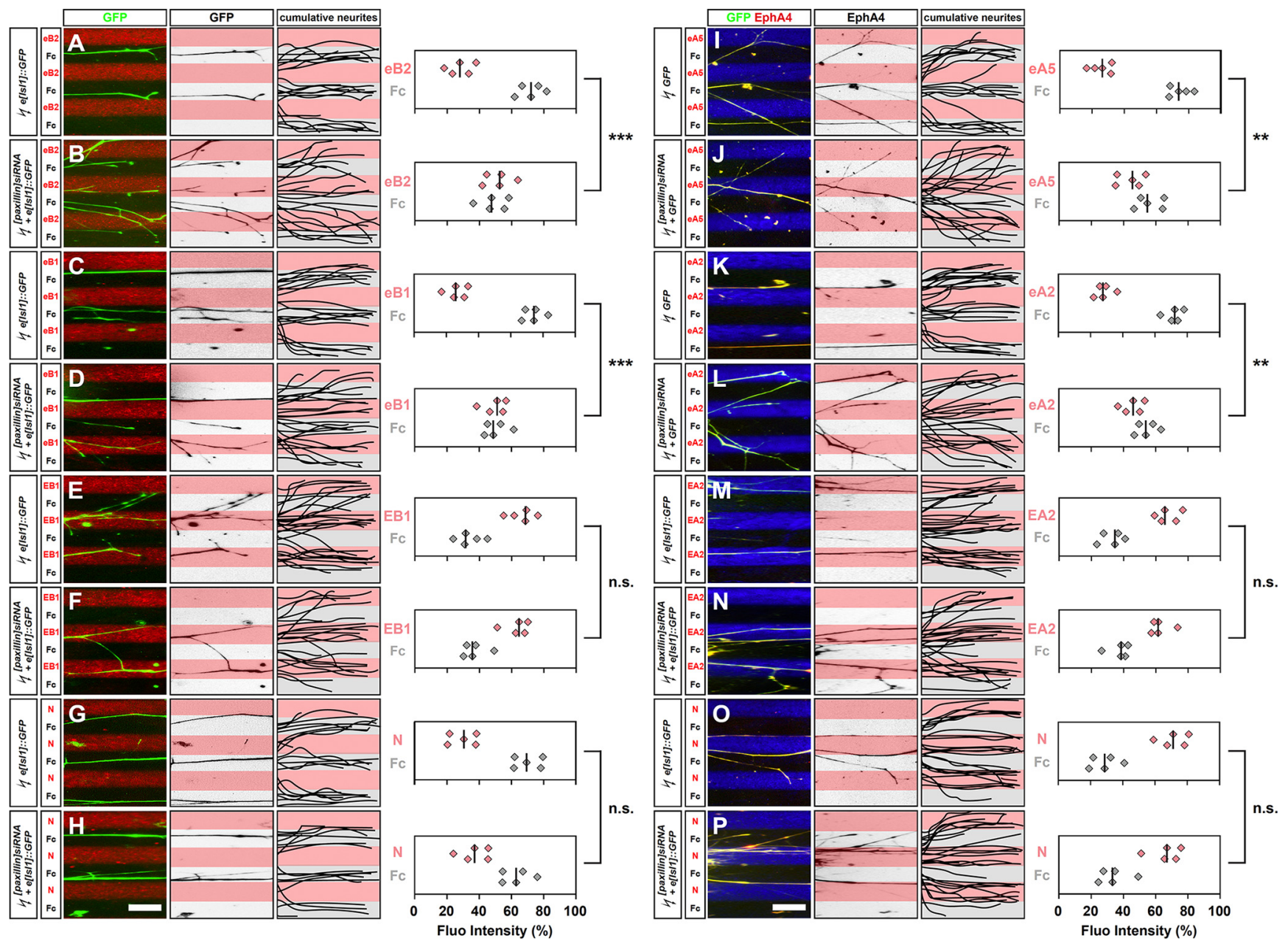

Figure 5. Paxillin function is specifically required for ephrin-mediated guidance of cultured LMC neurites. Growth preference on protein stripes exhibited by medial or lateral LMC axons. Each experiment is composed of three panels (left, middle, and right) and one quantification. $\boldsymbol{A}-\boldsymbol{H}$, Left panels, Detection of medial $\left(G F P^{+}\right)$LMC neurites of explants on eB2/Fc $(\boldsymbol{A})$, eB1/FC $(\boldsymbol{C})$, EB1/Fc $(\boldsymbol{E})$, or N/Fc $(\boldsymbol{G})$ stripes, and [paxillin]siRNA coelectroporated explants on eB2/Fc $(\boldsymbol{B})$, eB1/Fc $(\boldsymbol{D}), \mathrm{EB} 1 / \mathrm{Fc}(\boldsymbol{F})$, or N/Fc $(\boldsymbol{H})$ stripes. Middle panels, Inverted images where GFP signals are dark pixels overlaid on substrate stripes. Right panels, Superimposed images of five representative explants from each experimental group, highlighting the distribution of medial LMC neurites. Quantification of medial $\left(\mathrm{GFP}^{+}\right.$) LMC neurites on first (pink) and second (pale) stripes expressed as a percentage of total GFP signals. Minimal numbers of neurites, 90 . Numbers of embryos: $n=5$ for all groups. Minimal numbers of explants per embryo, 3. I-P, Left panels, Detection of lateral (GFP ${ }^{+}$EphA4 $\left.^{+}\right)$LMC neurites of explants on eA5/Fc $(\boldsymbol{I}), \mathrm{eA2} / \mathrm{Fc}(\boldsymbol{K})$, EA2/Fc $(\boldsymbol{M})$, or N/Fc (0) stripes, and [paxillin]siRNA coelectroporated explants on eA5/Fc $(\boldsymbol{J})$, eA2/Fc $(\boldsymbol{L}), \mathrm{EA2} / \mathrm{Fc}(\boldsymbol{N})$, or N/Fc $(\boldsymbol{P})$ stripes. Middle panels, Inverted images where EphA4 signals are dark pixel overlaid on substrate stripes. Right panels, Superimposed images of five representative explants from each experimental group, highlighting the distribution of lateral LMC neurites. Quantification of lateral (EphA4 ${ }^{+}$) LMC neurites on first (pink) and second (pale) stripes expressed as a percentage of total EphA4 signals. Minimal numbers of neurites, 88 . Numbers of embryos: $n=5$ for all groups. Minimal numbers of explants per embryo, 3. eB2, Ephrin-B2-Fc; eB1, ephrin-B1-Fc; EB1, EphB1-Fc; eA5, ephrin-A5-Fc; eA2, ephrin-A2-Fc; EA2, EphA2-Fc; N, Netrin-1. ${ }^{* * *} p<0.001$; ${ }^{* *} p<0.01 ; n$.s. $=$ not significant; statistical significance was computed using Mann-Whitney $U$ test; solid lines in each scatterplot show the median difference. Scale bars: $\boldsymbol{H}$ (for $\boldsymbol{A}-\boldsymbol{H}$ ), $\boldsymbol{P}$ (for I-P), $150 \mu \mathrm{m}$.

Medial LMC neurons coelectroporated with [paxillin]siRNA and $e[$ Isl1]::GFP exhibited significantly attenuated repulsion from ephrin-B2 and ephrin-B1 stripes compared with controls expressing $e[I s l 1]:: G F P$ only, suggesting that paxillin is required for EphB-mediated medial LMC axon repulsion from ephrin-B2 (Fig. $5 A, B ; p=5.41{ }^{*} 10^{-4}$ ) and ephrin-B1 (Fig. $5 C, D ; p=7.66$ * $\left.10^{-4}\right)$. Because of previous findings showing Eph-ephrin reverse signaling and Netrin-1 signaling as other pathways to modulate LMC axon trajectory into the limb (Marquardt et al., 2005; Dudanova et al., 2012; Luxey et al., 2015; Poliak et al., 2015), we also tested the response of medial LMC axons to stripes of EphB1 and Netrin-1 proteins in the context of paxillin loss-offunction. In contrast to those challenged with ephrin-B2 stripes, medial LMC neurons coelectroporated with [paxillin]siRNA and e[Isl1]::GFP exhibited a normal preference of EphB1 stripes compared with controls expressing e[Isl1]::GFP only (Fig. 5E,F; $p=0.3095$ ), implying a dispensable role of paxillin in EphB-
ephrinB reverse signaling in this context. Paxillin knockdown in medial LMC neurons also exhibited a normal avoidance of Netrin-1 stripes compared with controls expressing e[Isl1]::GFP only (Fig. 5G,H; $p=0.1925$ ), suggesting that paxillin does not play an important role in Netrin-1-mediated medial LMC axon guidance in vitro.

On the other hand, lateral LMC neurons coelectroporated with [paxillin]siRNA and GFP exhibited a significantly attenuated repulsion from ephrin-A5 and ephrin-A2 stripes compared with controls expressing GFP only, suggesting that paxillin is required for EphA-mediated lateral LMC axon repulsion from ephrin-A5 (Fig. 5I,J; $p=2.35{ }^{*} 10^{-3}$ ) and ephrin-A2 (Fig. $5 K, L$; $\left.p=6.08 * 10^{-3}\right)$. Next, we tested the response of lateral LMC axons to stripes of EphA2 and Netrin-1 proteins and observed that lateral LMC neurons coelectroporated with [paxillin]siRNA and GFP exhibited normal neurite attractive preference on EphA2 (Fig. $5 M, N ; p=0.2532$ ) or Netrin-1 stripes (Fig. 5O,P; 
$p=0.2177)$ compared with controls expressing GFP only, suggesting that, similar to medial LMC axons, paxillin does not play an important role in EphAephrinA or Netrin-1-mediated lateral LMC axon guidance in vitro. Altogether, these results demonstrate that paxillin is specifically required for ephrin-A- and ephrin-B-mediated LMC axon responses.

\section{Paxillin is required for Eph-mediated LMC motor axon guidance}

Both EphA4 and EphB2 receptors are detected in LMC subpopulations and are suggested to mediate lateral and medial LMC axon pathfinding, respectively (Henkemeyer et al., 1994; Soans et al., 1994; Kania and Jessell, 2003; Luria et al., 2008). To ascertain the role of paxillin in Eph signal transduction in vivo, we tested whether paxillin loss can attenuate the axon redirectional effects of Eph overexpression (Kania and Jessell, 2003; Luria et al., 2008). To this end, we coelectroporated [paxillin]siRNA with the Eph receptor GFP fusion protein expression plasmids EphA4::GFP or EphB2::GFP and compared with control EphA4::GFP and EphB2::GFP plasmid electroporations. EphA4::GFP electroporation in control embryos, as previously demonstrated (Eberhart et al., 2002; Kania and Jessell, 2003), induced a robust redirection of LMC axons into the dorsal limb nerves (Fig. 6A). In embryos coelectroporated with [paxillin]siRNA and EphA4:: $G F P$, the proportion of axonal GFP levels in ventral branches was significantly increased compared with EphA4::GFP electroporated controls (Fig. $6 A, B ; p=1.58^{\star} 10^{-3}$ ), indicating that paxillin knockdown attenuates EphA4-induced LMC motor axon redirection. In contrast, in embryos electroporated with EphB2:: GFP alone, a higher proportion of $\mathrm{GFP}^{+}$axons was observed in ventral LMC axons, suggesting that EphB2 overexpression is sufficient to redirect LMC axons into the ventral limb (Fig. 6C; Kao et al., 2009). In embryos coelectroporated with [paxillin]siRNA and EphB2::GFP, a significantly higher proportion of GFP levels was observed in the dorsal nerve branches compared with EphB2:: GFP alone, thus indicating that paxillin knockdown attenuates EphB2-induced LMC motor axon redirection (Fig. 6C,D; $p=7.25$ * $\left.10^{-4}\right)$. Together, these observations show that, in vivo, paxillin loss attenuates the LMC axon redirection induced by the Eph gain-offunction, suggesting that paxillin participates in Eph signaling in vivo.

\section{Paxillin is located downstream of Src in Eph signal} transduction during LMC pathfinding

Our previous work demonstrated that two Eph-signaling intermediaries, including Src and ephexin1, were involved in Eph-mediated LMC pathfinding (Kao et al., 2009; Chang et al., 2018). Src and ephexin1, like paxillin, are differentially required in LMC subdivisions: both Src and ephexin1 are heavily involved in EphB-mediated medial LMC pathfinding, and are only partially involved in EphAmediated lateral LMC pathfinding. Previous studies also suggested paxillin as a potential target of Src tyrosine phosphorylation (Weng et al., 1993; Schaller and Parsons, 1995; Carragher et al., 1999; Webb et al., 2004; Zaidel-Bar et al., 2007). Therefore, we examined the interaction of Src and ephexin1 with paxillin in EphB signal transduction during medial LMC motor axon pathfinding, where these molecules are potentially required. We first investigated whether paxillin is required for Src-mediated repulsion of medial LMC axons from ephrin-B2. Medial LMC neurons coexpressing e[Isl1]::GFP and Src expression plasmids showed significantly higher levels of repulsion from ephrin-B2 stripes compared with controls expressing e[Isl1]:: GFP alone, which confirmed that $\mathrm{Src}$ is required for EphB-mediated medial LMC axon repulsion from ephrin-B2 (Fig. $7 A, B ; p=0.0223$; Kao et al., 2009). We then coelectroporated e[Isl1]::GFP with an Src expression plasmid and [paxillin]siRNA and compared the effects to $e[$ Isl1]::GFP and Src plasmid coelectroporation. Medial LMC neurons coexpressing e[Isl1]::GFP, Src, and [paxillin]siRNA exhibited a significantly attenuated repulsion from ephrin-B2 stripes compared with $e$ [Isl1]::GFP and Src plasmid coelectroporation (Fig. $7 B, C ; p=4.15$ * $10^{-4}$ ). These observations suggest that paxillin acts downstream of Src in EphB signaling to mediate the repulsion of medial LMC axons from ephrin-B2.

To further investigate Src and paxillin interactions, in this context, we examined whether paxillin activation relies on Src; we used a paxillin ${ }^{Y 31 F / Y 118 F}$ expression plasmid, bearing an inactive mutant paxillin form that can no longer be phosphorylated by Src family kinases (Guan et al., 2007). Compared with GFP controls, LMC axon outgrowth and neuron specification and survival were normal in paxillin ${ }^{Y 31 F / Y 118 F}$ and GFP coexpressing embryos (Fig. $2 H, J-M ; p=0.5034$ for the ratio of $\mathrm{GFP}^{+}$axon length; $p=0.7638$ for total Foxp $1^{+}$neuron numbers/section; $p=0.2767$ for the proportions of lateral or medial LMC neurons), and $\mathrm{GFP}^{+}$LMC neurons indicated similar numbers of electroporated cells in both LMC divisions (Fig. 2H,J,N; $p=0.5820)$. As expected, medial LMC neurons coexpressing $e$ [Isl1]::GFP and paxillin expression plasmids showed significantly 

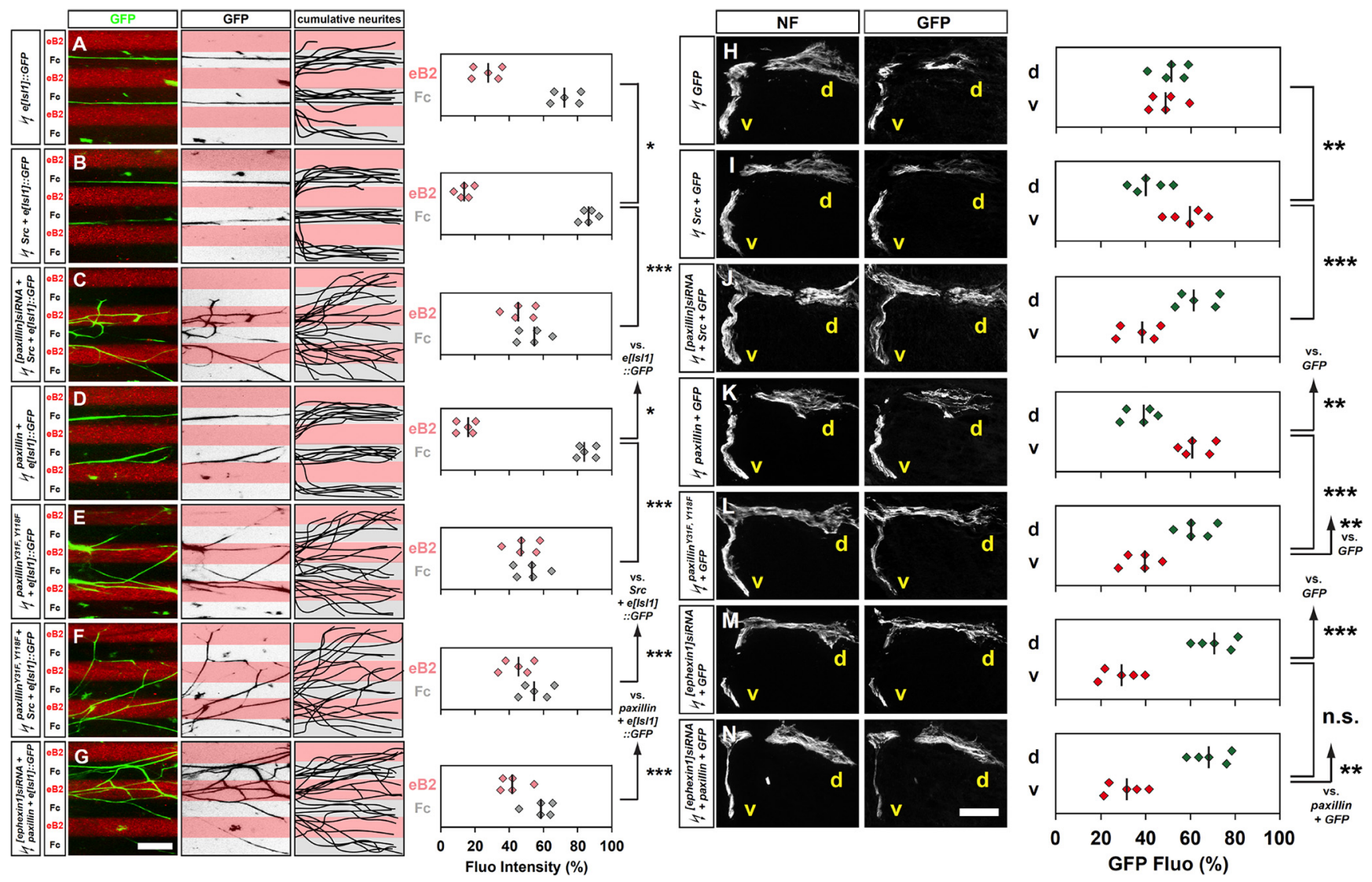

Figure 7. Paxillin mediates LMC motor axon pathfinding as an essential Eph intermediary functioning between Src and ephexin1. $A-G$, Growth preference on protein stripes exhibited by medial LMC axons. Each experiment is composed of three panels (left, middle, and right) and one quantification. Left panels, Detection of e[Is/1]::GFP (A), Src and e[Is/1]]::GFP (B), [paxillin]

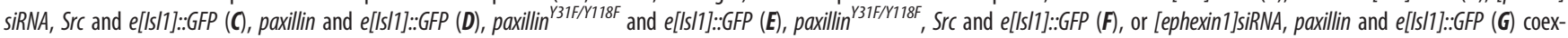
pressed medial $\left(G_{F P}^{+}\right)$LMC neurites of explants on eB2/Fc stripes. Middle panels, Inverted images where GFP signals are dark pixel overlaid on substrate stripes. Right panels, Superimposed images of five representative explants from each experimental group, highlighting the distribution of medial LMC neurites. Quantification of medial (GFP ${ }^{+}$) LMC neurites on first (pink) and second (pale) stripes expressed as a percentage of total GFP signals. Minimal numbers of neurites, 90 . Numbers of embryos: $n=5$ for all groups. Minimal numbers of explants per embryo, 3 . $\boldsymbol{H -}$ $\boldsymbol{N}$, All images are from chick HH st. 28/29 lumbar levels. Neurofilament and GFP detection in the limb nerve branches in the crural plexus of chick embryos electroporated with the following

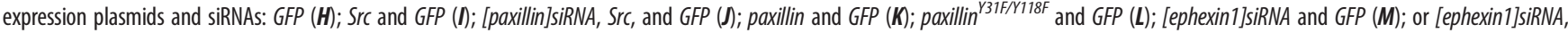
paxillin, and GFP $(\boldsymbol{N})$. Quantification of GFP signals in all electroporation experiments expressed as, respectively, the percentage in dorsal and ventral limb nerves [GFP Fluo (\%)]. Numbers of embryos: $n=5$ for all groups. eB2, Ephrin-B2-Fc; d, dorsal; v, ventral. ${ }^{* * *} p<0.001 ;{ }^{* *} p<0.01 ;{ }^{*} p<0.05 ; n . s$. = not significant; statistical significance computed using Mann-Whitney $U$ test; solid lines in each scatterplot show the median difference. Scale bars: $\mathbf{G}$ (for $\boldsymbol{A}-\boldsymbol{G}), \boldsymbol{N}$ (for $\boldsymbol{H}-\boldsymbol{M}), 150 \mu \mathrm{m}$.

higher levels of repulsion from ephrin-B2 stripes compared with $e[I s l 1]:: G F P$ controls (Fig. $7 A, D ; p=0.0274$ ), while medial LMC neurons coexpressing paxillin ${ }^{Y 31 F / Y 118 F}$ and $e[I s l 1]:: G F P$ exhibited significantly attenuated repulsion from ephrin-B2 stripes compared with paxillin and e[Isl1]::GFP coelectroporation (Fig. $\left.7 D, E ; p=3.23 * 10^{-5}\right)$. In addition, medial LMC neurons coexpressing e[Isl1]::GFP, Src, and paxillin ${ }^{Y 31 F / Y 118 F}$ exhibited significantly attenuated repulsion from ephrin-B2 stripes compared with those expressing e[Isl1]::GFP and $S r c$ only (Fig. $7 B, F ; p=5.39 * 10^{-5}$ ). Together, these results confirm that paxillin phosphorylation by Src contributes to EphBmediated medial LMC axon repulsion from ephrin-B2. Next, to test the possibility of ephexin 1 as a downstream target of paxillin, we investigated whether ephexin 1 is required for paxillin-mediated repulsion of medial LMC axons from ephrin-B2. Medial LMC neurons coexpressing [ephexin1]siRNA, paxillin, and $e[$ Isl1]::GFP exhibited significantly attenuated repulsion from ephrin-B2 stripes compared with paxillin and $e\left[\right.$ Isl1]::GFP electroporation (Fig. 7D,G; $p=7.67 * 10^{-5}$ ). These observations thus suggest that ephexin 1 acts downstream of paxillin in EphB signaling to mediate the repulsion of medial LMC axons from ephrin-B2.
To further investigate this hypothesis in vivo, we knocked down paxillin using [paxillin]siRNA and checked whether the Src-induced LMC motor axon redirection was affected. As previously shown (Kao et al., 2009), in embryos coelectroporated with Src and GFP, a significantly higher proportion of $\mathrm{GFP}^{+}$axons was observed in the ventral nerve branches when compared with GFP controls (Fig. $7 H, I ; p=4.54 * 10^{-3}$ ), suggesting that Src overexpression leads to a significantly higher proportion of LMC motor axons accessing the ventral limb. In contrast, in embryos coelectroporated with Src, GFP, and [paxillin]siRNA, a significantly higher proportion of $\mathrm{GFP}^{+}$axons was observed in the dorsal limb nerve compared with $S r c$ and GFP coexpression (Fig. $\left.7 I, J ; p=3.39{ }^{*} 10^{-4}\right)$, which indicates that paxillin knockdown attenuates Src-induced LMC motor axon redirection. In addition, in embryos coelectroporated with paxillin ${ }^{Y 31 F / Y 118 F}$ and GFP expression plasmids, a significantly higher proportion of $\mathrm{GFP}^{+}$axons was observed in the dorsal nerve branches compared with both GFP controls, and paxillin and GFP coelectroporation (Fig. $7 H, K, L ; p=3.11^{\star} 10^{-3}$ vs $G F P, p=2.65 * 10^{-4}$ vs paxillin + GFP), which confirmed the requirement of paxillin phosphorylation by Src for LMC axon pathfinding. Next, we knocked down ephexin1 using [ephexin1]siRNA, and, as 
expected, a significantly higher proportion of $\mathrm{GFP}^{+}$axons was observed in the dorsal nerve branches when compared with GFP controls (Fig. $7 H, M ; p=6.02 * 10^{-4}$ ). Similarly, in embryos coelectroporated with [ephexin1] siRNA, paxillin, and GFP, a significantly higher proportion of $\mathrm{GFP}^{+}$axons was observed in the dorsal nerve branches when compared with paxillin and GFP coexpression (Fig. $7 K, N, p=1.88{ }^{\star} 10^{-3}$; Fig. $7 M, N, p=0.3623$ ), suggesting that ephexin1 knockdown, with or without ectopic paxillin expression, significantly increases the proportion of LMC motor axons entering the dorsal limb. Together, these results suggest that paxillin plays essential Eph-signaling intermediary functions downstream of Src, but upstream of ephexin1 to modulate the limb trajectory of LMC motor axons.

\section{Discussion}

Paxillin has been proposed to function in signaling pathways essential for cytoskeletal rearrangement, including early neuronal differentiation and migration. Consequently, we investigated its potential roles in axon pathfinding in vivo and demonstrated that paxillin is essential for the fidelity of motor axon trajectory selection in the limb. Herein, we discuss the role of paxillin in axon outgrowth and neuronal survival and migration, the differential requirement of paxillin function by LMC subpopulations, as well as its implications in Eph signal relay.

Paxillin is essential for motor axon guidance but dispensable for outgrowth and neuronal survival and migration

Paxillin has been proposed to modulate neurite formation and extension: paxillin tyrosine phosphorylation mediates F-actin redistribution during cell spread and nerite formation (Leventhal and Feldman, 1996). Independent in vitro studies also indicate that the maintenance of intracellular paxillin levels by adaptor protein Nck2 in vitro is involved in neuritogenesis (Guan et al., 2007), and paxillin phosphorylation by p38 MAPK mediates NGF-induced neurite outgrowth (Ivankovic-Dikic et al., 2000; Huang et al., 2004). Interestingly, we did not observe changes in limb nerve axon outgrowth in chick embryos with attenuated or enhanced paxillin expression in LMC neurons. Although we cannot exclude the possibility that the residual paxillin expression following siRNA knockdown is sufficient to mediate axon outgrowth, paxillin activity appears to have a limited effect on motor axon outgrowth. A reasonable explanation is that different neuronal types express different repertoires of paxillin targets and interact with different kinetics and affinities with guidance receptors. Alternatively, each cell type may respond differently to the same paxillin binding partners, such as FAK, which mediates the responses of some neuronal types toward repellant cues, but are also involved in cell spreading and growth cone attraction in other cell types (Carter et al., 2002; Li et al., 2004; Liu et al., 2004; Ren et al., 2004; Guan et al., 2007; Bechara et al., 2008; Woo et al., 2009; Chacón et al., 2010; Myers and Gomez, 2011; Kerstein et al., 2017).

In addition to axonal remodeling, paxillin has been suggested to mediate cell survival and migration (Turner, 1991; Turner et al., 1993; Price et al., 1998; Chay et al., 2002; Brown and Turner, 2004; Melendez et al., 2004; Sorenson, 2004; Subauste et al., 2004; Sheibani et al., 2008). However, we did not observe obvious changes in either numbers or positions of LMC neurons with modified paxillin expression in chick or mouse embryos. Recent studies using neural-specific paxillin deletion (Nes-Cre: $P x n^{F / F}$ ) also showed no obvious changes in the numbers of cortical neurons in the developing cortex, despite the overall slower cortical neuron migration speed leading to delay in deep cortical layer formation prenatally and perinatally, but is no longer detected in adulthood (Rashid et al., 2017). It is thus possible that the role of paxillin in cell survival is redundant in the CNS, and that its involvement in cell migration is only stage dependent or limited. In addition to the possible explanations above, a thorough investigation of the expression pattern of other focal adhesion kinase members in different stages of spinal motor neuron differentiation could potentially clarify these issues.

\section{The differential requirement of paxillin function by medial and lateral LMC neurons}

Paxillin is expressed in most LMC neurons and is required for proper axon trajectory of both medial and lateral LMC divisions, suggesting a nonredundant role in LMC axon pathfinding. This raises the question of how paxillin relays guidance signals to the cytoskeleton. Our data showing loss of paxillin-induced axon redirection following ephexin1 knockdown, a RhoGEF (guanine nucleotide exchange factor), imply that paxillin mediates RhoGTPase activity via ephexin1. Indeed, since paxillin was identified as a major component of focal adhesions, it has emerged as a key coordinator of RhoGTPase activity by recruitment of GEFs and GAPs (GTPase-activating proteins), such as DOCK180, CrkII, and p190RhoGAP (Price et al., 1998; Petit et al., 2000; Brugnera et al., 2002; Tsubouchi et al., 2002; Brown and Turner, 2004; Grimsley et al., 2004). On the other hand, various biochemical evidences imply that paxillin activity is enhanced via Src during different cellular events (Weng et al., 1993; Schaller and Parsons, 1995; Carragher et al., 1999; Webb et al., 2004; Zaidel-Bar et al., 2007). Thus, a general model of paxillin function in axon guidance could first involve Src recruited by the $\mathrm{C}$-terminal domain of a guidance receptor activated by its ligand, followed by phosphorylation of Src family kinase targets including paxillin. Subsequently, ephexin1 activation by paxillin is required for small GTPase activity resulting in growth cone turning, as shown by our observations of misprojecting spinal motor axons in paxillin mutants. Indeed, our data showing the attenuation of Src-mediated motor axon redirection by paxillin loss, the ectopic expression of nonphosphorylable paxillin, and the attenuation of paxillin-mediated redirection by ephexin1 loss, indicate that paxillin is a target of Src regulating ephexin 1 activity in this context.

Our data indicate that although paxillin is expressed in most LMC neurons, its requirement in the fidelity of LMC axon trajectory selection appears to be differential; medial LMC pathfinding appears to depend on paxillin function to a greater extent than lateral LMC pathfinding. This differential requirement of paxillin could be because of multiple pathways being required for the guidance of either LMC division. Strong evidence has demonstrated EphA and EphB signaling in the guidance of lateral and medial LMC axons, respectively (Kania and Jessell, 2003; Luria et al., 2008). The differential sensitivity of lateral and medial LMC neurons to paxillin could thus reflect the differential requirement of paxillin in EphA and EphB receptor signaling (for more details, see subsection Paxillin function in ephrin-Eph signaling). Previous findings also suggest Netrin-1 as a bifunctional ligand, attracting lateral LMC and repelling medial LMC axons (Poliak et al., 2015). In addition, several ancillary systems including GDNF:c-Ret and Semaphorin3F:Neuropilin2 are proposed to mediate proper trajectory of motor axons from LMC subpopulations (Huber et al., 2005; Kramer et al., 2006; Moret et al., 2007; Dudanova et al., 2010). There is still no biochemical evidence of Netrin-1 receptors (Dcc and Unc5c), c-Ret, or Neuropilin2 association with paxillin. However, our in vitro data showing no 
obvious changes in LMC neurite growth preferences toward Netrin-1 stripes in a paxillin knockdown suggest a dispensable role of paxillin in Netrin-1-mediated LMC pathfinding. To thoroughly investigate the involvement of paxillin in other guidance systems in this context, in vitro responses to individual cues such as GDNF or semaphorins using similar approaches could be measured in cultured motor neurons lacking paxillin (Huber et al., 2005; Kramer et al., 2006; Dudanova et al., 2010; Bonanomi et al., 2012).

\section{Paxillin function in ephrin-Eph signaling}

Our experiments implicating paxillin in ephrin-Eph forward signaling are in line with previous in vitro studies suggesting a potential role of paxillin in EphB-mediated cell migration (Vindis et al., 2004). In addition, other studies also demonstrated paxillin phosphorylation following stimulation of EphA2 with ephrin-A1 in renal tubular epithelial cells, implying paxillin function in ephrin-A-EphA signaling (Baldwin et al., 2006). Our data showing differential paxillin requirements in Eph-mediated motor axon guidance suggest that paxillin plays an essential role in EphB-mediated medial LMC pathfinding, and only a partial role in EphA-mediated lateral LMC pathfinding. Combined with previous findings showing the differential requirement of Src in Eph-mediated LMC guidance (Kao et al., 2009), our data showing paxillin as a potential target of Src suggest that paxillin functions as an essential intermediary downstream of Src, which can modulate ephrin-Eph signaling in LMC pathfinding. However, when comparing our in vitro data showing a dispensable role of paxillin in Netrin-1-mediated LMC axon trajectory selection with recent studies showing Src function in both Eph and Netrin-1 signaling in LMC neurons, we propose that there are Src targets other than paxillin required in guidance pathways other than ephrin-Eph (Poliak et al., 2015). Other effectors such as FAK are also implicated in both Eph pathways, implying that, if present, they could relay Src signals downstream of multiple guidance receptors (Miao et al., 2000; Carter et al., 2002; Vindis et al., 2004).

In addition to the repulsive ephrin-Eph forward signaling, the attractive Eph-ephrin reverse signaling has been proposed to function as an ancillary guidance system to ensure the proper trajectory of LMC axons into the limbs (Marquardt et al., 2005; Kao and Kania, 2011; Dudanova et al., 2012; Luxey et al., 2015). Our in vitro data showing a dispensable role of paxillin in Ephephrin signaling thus imply the requirement of different sets of intermediaries downstream of ephrin-Eph versus Eph-ephrin signaling during LMC pathfinding. Further investigation of the involvement of some potential Eph-ephrin effectors, such as Grb4 and Fyn, should clarify the Eph-ephrin pathway in this context (Davy et al., 1999; Cowan and Henkemeyer, 2001).

Two reasonable explanations for the partial paxillin requirement in lateral LMC neurons are that paxillin is only required for the guidance of a lateral LMC subpopulation, or that paxillin is redundant with other EphA effectors participating in lateral LMC axon guidance, for instance, other Rho family GEFs including Kalirin and Vav2, are also implicated as Eph effectors. Kalirin is preferentially required as an EphB but not as an EphA effector to modulate cytoskeletal dynamics, but Vav2 can interact with both Eph pathways to modulate growth cone motility (Penzes et al., 2003; Moeller et al., 2006). Further investigation of Vav2 expression and function in LMC could thus provide a better understanding of either Eph pathway in LMC pathfinding.

\section{Conclusion}

In this study, we have shown that paxillin is required for motor axon trajectory selection in vivo. This is the first report pointing to an important role of paxillin in Eph signaling, and directly comparing its involvement in EphA- versus EphB-regulated motor axon guidance to show a differential requirement of paxillin in different Eph pathways in vivo, thus highlighting the importance of the LMC motor axon projection system as a model of simple axon guidance decision where the role of the ephrinEph signaling relay machinery can be investigated.

\section{References}

Baldwin C, Chen ZW, Bedirian A, Yokota N, Nasr SH, Rabb H, Lemay S (2006) Upregulation of EphA2 during in vivo and in vitro renal ischemia-reperfusion injury: role of Src kinases. Am J Physiol Renal Physiol 291:F960-F971.

Bechara A, Nawabi H, Moret F, Yaron A, Weaver E, Bozon M, Abouzid K, Guan JL, Tessier-Lavigne M, Lemmon V, Castellani V (2008) FAKMAPK-dependent adhesion disassembly downstream of L1 contributes to semaphorin3A-induced collapse. EMBO J 27:1549- 1562.

Bonanomi D, Chivatakarn O, Bai G, Abdesselem H, Lettieri K, Marquardt T, Pierchala BA, Pfaff SL (2012) Ret is a multifunctional coreceptor that integrates diffusible- and contact-axon guidance signals. Cell 148:568582.

Brown MC, Turner CE (2004) Paxillin: adapting to change. Physiol Rev 84:1315-1339.

Brugnera E, Haney L, Grimsley C, Lu M, Walk SF, Tosello-Trampont AC, Macara IG, Madhani H, Fink GR, Ravichandran KS (2002) Unconventional Rac-GEF activity is mediated through the Dock180ELMO complex. Nat Cell Biol 4:574-582.

Carragher NO, Levkau B, Ross R, Raines EW (1999) Degraded collagen fragments promote rapid disassembly of smooth muscle focal adhesions that correlates with cleavage of pp125(FAK), paxillin, and talin. J Cell Biol 147:619-630.

Carter N, Nakamoto T, Hirai H, Hunter T (2002) EphrinA1-induced cytoskeletal re-organization requires FAK and p130 ${ }^{\text {cas }}$. Nat Cell Biol 4:565573.

Chacón MR, Fernández G, Rico B (2010) Focal adhesion kinase functions downstream of Sema3A signaling during axonal remodeling. Mol Cell Neurosci 44:30-42.

Chang C-J, Chang M-Y, Chou S-Y, Huang C-C, Chuang J-Y, Hsu T-I, Chang H-F, Wu Y-H, Wu C-C, Morales D, Kania A, Kao T-J (2018) Ephexin1 is required for Eph-mediated limb trajectory of spinal motor axons. J Neurosci 38:2043-2056.

Chay KO, Park SS, Mushinski JF (2002) Linkage of caspase-mediated degradation of paxillin to apoptosis in $\mathrm{Ba} / \mathrm{F} 3$ murine pro-B lymphocytes. J Biol Chem 277:14521-14529.

Cowan CA, Henkemeyer M (2001) The SH2/SH3 adaptor Grb4 transduces B-ephrin reverse signals. Nature 413:174-179.

Davy A, Gale NW, Murray EW, Klinghoffer RA, Soriano P, Feuerstein C, Robbins SM (1999) Compartmentalized signaling by GPI-anchored ephrin-A5 requires the Fyn tyrosine kinase to regulate cellular adhesion. Genes Dev 13:3125-3135.

Deakin N, Turner CE (2008) Paxillin comes of age. J Cell Sci 121:2435-2444.

Dudanova I, Gatto G, Klein R (2010) GDNF acts as a chemoattractant to support ephrinA-induced repulsion of limb motor axons. Curr Biol 20:21502156.

Dudanova I, Kao TJ, Herrmann JE, Zheng B, Kania A, Klein R (2012) Genetic evidence for a contribution of EphA:ephrinA reverse signaling to motor axon guidance. J Neurosci 32:5209-5215.

Eberhart J, Swartz ME, Koblar SA, Pasquale EB, Krull CE (2002) EphA4 constitutes a population-specific guidance cue for motor neurons. Dev Biol 247:89- 101

Gallarda BW, Bonanomi D, Müller D, Brown A, Alaynick WA, Andrews SE, Lemke G, Pfaff SL, Marquardt T (2008) Segregation of axial motor and sensory pathways via heterotypic trans-axonal signaling. Science 320:233-236.

Grimsley CM, Kinchen JM, Tosello-Trampont AC, Brugnera E, Haney LB, Lu M, Chen Q, Klingele D, Hengartner MO, Ravichandran KS (2004) 
Dock180 and ELMO1 proteins cooperate to promote evolutionarily conserved Rac-dependent cell migration. J Biol Chem 279:6087-6097.

Guan S, Chen M, Woodley D, Li W (2007) Nck $\beta$ adapter controls neuritogenesis by maintaining the cellular paxillin level. Mol Cell Biol 27:60016011.

Hagel M, George EL, Kim A, Tamimi R, Opitz SL, Turner CE, Imamoto A, Thomas SM (2002) The adaptor protein paxillin is essential for normal development in the mouse and is a critical transducer of fibronectin signaling. Mol Cell Biol 22:901-915.

Hamburger V, Hamilton HL (1951) A series of normal stages in the development of the chick embryo. J Morphol 88:195-272.

Helmbacher F, Schneider-Maunoury S, Topilko P, Tiret L, Charnay P (2000) Targeting of the EphA4 tyrosine kinase receptor affects dorsal/ventral pathfinding of limb motor axons. Development 127:3313-3324.

Henkemeyer M, Marengere LEM, McGlade J, Olivier JP, Conlon RA, Holmyard DP, Letwin K, Pawson T (1994) Immunolocalization of the Nuk receptor tyrosine kinase suggests roles in segmental patterning of the brain and axonogenesis. Oncogene 9:1001-1014.

Huang C, Borchers CH, Schaller MD, Jacobson K (2004) Phosphorylation of paxillin by $\mathrm{p} 38 \mathrm{MAPK}$ is involved in the neurite extension of PC-12 cells. J Cell Biol 164:593-602.

Huber AB, Kania A, Tran TS, Gu C, De Marco Garcia N, Lieberam I, Johnson D, Jessell TM, Ginty DD, Kolodkin AL (2005) Distinct roles for secreted semaphorin signaling in spinal motor axon guidance. Neuron 48:949-964.

Ivankovic-Dikic I, Grönroos E, Blaukat A, Barth BU, Dikic I (2000) Pyk2 and FAK regulate neurite outgrowth induced by growth factors and integrins. Nat Cell Biol 2:574-581.

Kania A, Jessell TM (2003) Topographic motor projections in the limb imposed by LIM homeodomain protein regulation of ephrin-A:ephA interactions. Neuron 38:581-596.

Kania A, Johnson RL, Jessell TM (2000) Coordinate roles for LIM homeobox genes in directing the dorsoventral trajectory of motor axons in the vertebrate limb. Cell 102:161-173.

Kao T-J, Kania A (2011) Ephrin-mediated cis-attenuation of Eph receptor signaling is essential for spinal motor axon guidance. Neuron 71:76-91.

Kao T-J, Palmesino E, Kania A (2009) SRC family kinases are required for limb trajectory selection by spinal motor axons. J Neurosci 29:56905700.

Kao T-J, Nicholl GC, Johansen JA, Kania A, Beg AA (2015) $\alpha 2$-chimaerin is required for Eph receptor-class-specific spinal motor axon guidance and coordinate activation of antagonistic muscles. J Neurosci 35:2344-2357.

Kerstein PC, Patel KM, Gomez TM (2017) Calpain-mediated proteolysis of talin and FAK regulates adhesion dynamics necessary for axon guidance. J Neurosci 37:1568-1580.

Knöll B, Drescher U (2004) Src family kinases are involved in EphA receptor-mediated retinal axon guidance. J Neurosci 24:6248-6257.

Knöll B, Weinl C, Nordheim A, Bonhoeffer F (2007) Stripe assay to examine axonal guidance and cell migration. Nat Protoc 2:1216-1224.

Kramer ER, Knott L, Su F, Dessaud E, Krull CE, Helmbacher F, Klein R (2006) Cooperation between GDNF/Ret and ephrinA/EphA4 signals for motor-axon pathway selection in the limb. Neuron 50:35-47.

Lance-Jones C, Landmesser L (1981) Pathway selection by embryonic chick motoneurons in an experimentally altered environment. Proc $\mathrm{R}$ Soc Lond B Biol Sci 214:19- 52.

Landmesser L (1978) The development of motor projection patterns in the chick hind limb. J Physiol 284:391-414.

Leventhal PS, Feldman EL (1996) Tyrosine phosphorylation and enhanced expression of paxillin during neuronal differentiation in vitro. J Biol Chem 271:5957-5960.

Li W, Lee J, Vikis HG, Lee SH, Liu G, Aurandt J, Shen TL, Fearon ER, Guan JL, Han M, Rao Y, Hong K, Guan KL (2004) Activation of FAK and Src are receptor-proximal events required for netrin signaling. Nat Neurosci 7:1213-1221.

Liu G, Beggs H, Jürgensen C, Park HT, Tang H, Gorski J, Jones KR, Reichardt LF, Wu J, Rao Y (2004) Netrin requires focal adhesion kinase and Src family kinases for axon outgrowth and attraction. Nat Neurosci 7:1222-1232.

Luria V, Laufer E (2007) Lateral motor column axons execute a ternary trajectory choice between limb and body tissues. Neural Dev 2:13.
Luria V, Krawchuk D, Jessell TM, Laufer E, Kania A (2008) Specification of motor axon trajectory by ephrin-B:ephB signaling: symmetrical control of axonal patterning in the developing limb. Neuron 60:1039- 1053.

Luxey M, Laussu J, Davy A (2015) EphrinB2 sharpens lateral motor column division in the developing spinal cord. Neural Dev 10:25.

Marquardt T, Shirasaki R, Ghosh S, Andrews SE, Carter N, Hunter T, Pfaff SL (2005) Coexpressed EphA receptors and ephrin-A ligands mediate opposing actions on growth cone navigation from distinct membrane domains. Cell 121:127-139.

Melendez J, Turner C, Avraham H, Steinberg SF, Schaefer E, Sussman MA (2004) Cardiomyocyte apoptosis triggered by RAFTK/pyk2 via Src kinase is antagonized by paxillin. J Biol Chem 279:53516-53523.

Miao H, Burnett E, Kinch M, Simon E, Wang BC (2000) Activation of EphA2 kinase suppresses integrin function and causes focal-adhesion-kinase dephosphorylation. Nat Cell Biol 2:62-69.

Miyamoto Y, Torii T, Yamamori N, Eguchi T, Nagao M, Nakamura K, Tanoue A, Yamauchi J (2012) Paxillin is the target of c-Jun N-terminal kinase in Schwann cells and regulates migration. Cell Signal 24:2016-2069.

Moeller ML, Shi Y, Reichardt LF, Ethell IM (2006) EphB receptors regulate dendritic spine morphogenesis through the recruitment/phosphorylation of focal adhesion kinase and RhoA activation. J Biol Chem 281:1587-1598.

Momose T, Tonegawa A, Takeuchi J, Ogawa H, Umesono K, Yasuda K (1999) Efficient targeting of gene expression in chick embryos by microelectroporation. Dev Growth Differ 41:335-344.

Moret F, Renaudot C, Bozon M, Castellani V (2007) Semaphorin and neuropilin co-expression in motoneurons sets axon sensitivity to environmental semaphorin sources during motor axon pathfinding. Development 134:4491-4501.

Myers JP, Gomez TM (2011) Focal adhesion kinase promotes integrin adhesion dynamics necessary for chemotropic turning of nerve growth cones. J Neurosci 31:13585-13595.

Penzes P, Beeser A, Chernoff J, Schiller MR, Eipper BA, Mains RE, Huganir RL (2003) Rapid induction of dendritic spine morphogenesis by transsynaptic EphrinB-EphB receptor activation of the Rho-GEF Kalirin. Neuron 37:263-274.

Petit V, Boyer B, Lentz D, Turner CE, Thiery JP, Vallés AM (2000) Phosphorylation of tyrosine residues 31 and 118 on paxillin regulates cell migration through an association with CRK in NBT-II cells. J Cell Biol 148:957-970.

Poliak S, Morales D, Croteau L-P, Krawchuk D, Palmesino E, Morton S, Cloutier J-F, Charron F, Dalva MB, Ackerman SL, Kao T-J, Kania A (2015) Synergistic integration of Netrin and ephrin axon guidance signals by spinal motor neurons. eLife 4:e10841.

Price LS, Leng J, Schwartz MA, Bokoch GM (1998) Activation of Rac and Cdc42 by integrins mediates cell spreading. Mol Biol Cell 9:1863-1871.

Rashid M, Belmont J, Carpenter D, Turner CE, Olson EC (2017) Neural-specific deletion of the focal adhesion adaptor protein paxillin slows migration speed and delays cortical layer formation. Development 144:40024014.

Ren XR, Ming GL, Xie Y, Hong Y, Sun DM, Zhao ZQ, Feng Z, Wang Q, Shim S, Chen ZF, Song HJ, Mei L, Xiong WC (2004) Focal adhesion kinase in netrin-1 signaling. Nat Neurosci 7:1204- 1212.

Rozen S, Skaletsky H (2000) Primer3 on the WWW for general users and for biologist programmers. Methods Mol Biol 132:365-386.

Schaeren-Wiemers N, Gerfin-Moser A (1993) A single protocol to detect transcripts of various types and expression levels in neural tissue and cultured cells: in situ hybridization using digoxigenin-labelled cRNA probes. Histochemistry 100:431-440.

Schaller MD, Parsons JT (1995) pp125FAK-dependent tyrosine phosphorylation of paxillin creates a high-affinity binding site for Crk. Mol Cell Biol 15:2635-2645

Sheibani N, Tang Y, Sorenson CM (2008) Paxillin's LD4 motif interacts with bcl-2. J Cell Physiol 214:655-661.

Soans C, Holash JA, Pasquale EB (1994) Characterization of the expression of the receptor-type tyrosine kinase during development and in tumor cell lines. Oncogene 9:3353-3361.

Sorenson CM (2004) Interaction of bcl-2 with Paxillin through its BH4 domain is important during ureteric bud branching. J Biol Chem 279:11368-11137.

Subauste MC, Pertz O, Adamson ED, Turner CE, Junger S, Hahn KM (2004) Vinculin modulation of paxillin-FAK interactions regulates ERK to control survival and motility. J Cell Biol 165:371-381. 
Tosney KW, Landmesser LT (1985) Development of the major pathways for neurite outgrowth in the chick hindlimb. Dev Biol 109:193-214.

Tsubouchi A, Sakakura J, Yagi R, Mazaki Y, Schaefer E, Yano H, Sabe H (2002) Localized suppression of RhoA activity by Tyr31/118-phosphorylated paxillin in cell adhesion and migration. J Cell Biol 159:673-683.

Tsuchida T, Ensini M, Morton SB, Baldassare M, Edlund T, Jessell TM, Pfaff SL (1994) Topographic organization of embryonic motor neurons defined by expression of LIM homeobox genes. Cell 79:957-970.

Turner CE (1991) Paxillin is a major phosphotyrosine-containing protein during embryonic development. J Cell Biol 115:201-207.

Turner CE, Glenney JR Jr, Burridge K (1990) Paxillin: a new vinculin binding protein present in focal adhesions. J Cell Biol 111:1059-1068.

Turner CE, Schaller MD, Parsons JT (1993) Tyrosine phosphorylation of the focal adhesion kinase pp125FAK during development: relation to paxillin. J Cell Sci 105:637-645.
Vindis C, Teli T, Cerretti DP, Turner CE, Huynh-Do U (2004) EphB1-mediated cell migration requires the phosphorylation of paxillin at Tyr-31/ Tyr-118. J Biol Chem 279:27965-27970.

Webb DJ, Donais K, Whitmore LA, Thomas SM, Turner CE, Parsons JT, Horwitz AF (2004) FAK-Src signalling through paxillin, ERK and MLCK regulates adhesion disassembly. Nat Cell Biol 6:154-161.

Weng Z, Taylor JA, Turner CE, Brugge JS, Seidel-Dugan C (1993) Detection of Src homology 3-binding proteins, including paxillin, in normal and vSrc transformed Balb/c 3T3 cells. J Biol Chem 268:14956-14963.

Woo S, Rowan DJ, Gomez TM (2009) Retinotopic mapping requires focal adhesion kinase-mediated regulation of growth cone adhesion. J Neurosci 29:13981-13991.

Zaidel-Bar R, Milo R, Kam Z, Geiger B (2007) A paxillin tyrosine phosphorylation switch regulates the assembly and form of cell-matrix adhesions. J Cell Sci 120:137-148. 